\title{
Diplopodes platydesmides et polyzonides collectés dans le Nord- ouest de la Péninsule Ibérique par les missions britanniques de 1993 et 2004 (Diplopoda : Platydesmida, Polyzoniida)
}

\author{
Platydesmidan and polyzoniidan millipedes collected in the \\ Northwest of Iberian Peninsula by British expeditions in 1993 and \\ 2004 (Diplopoda: Platydesmida, Polyzoniida)
}

\author{
Авупарноногие многоножкии-пиатидесмиды и полизонииды, \\ собранные на северо-западе Иберийского полуострова \\ британскими экспедиџиями в 1993 и 2004 годах \\ (Diplopoda: Platydesmida, Polyzoniida)
}

\author{
Jean-Paul Mauriès \\ Жан-Поль Морьес
}

\begin{abstract}
Attaché honoraire au Muséum National d'Histoire Naturelle, Dpt. Systématique et Evolution, CP53, 61 rue Buffon, 75231 Paris Cedex 05 , France. E-mail : mauries.jean-paul@wanadoo.fr
\end{abstract}

MOTS-CLÉS : Diplopoda, Platydesmida, Polyzoniida, taxonomie, genre nouveau, espèce nouvelle, clé de determination, carte, Espagne, Portugal.

KEY WORDS: Diplopoda, Platydesmida, Polyzoniida, taxonomy, new genus, new species, identification key, map, Spain, Portugal.

КЛЮЧЕВЫЕ СЛОВА: Diplopoda, Platydesmida, Polyzoniida, таксономия, новый род, новый вид, ключ, карта, Испания, Португалия.

RÉSUMÉ. Les résultats de l'identification des diplopodes platydesmides et polyzonides édaphiques collectés par les missions britanniques de 1993 et 2004 dans le Nord-ouest de la Péninsule Ibérique (Galice et extrême nord du Portugal) sont présentés. Un Polyzoniida du genre Hirudisoma et deux espèces nouvelles de Platydesmida sont décrites, appartenant l'une au genre Dolistenus, D. iberoalbus sp.n., l'autre à un nouveau genre monospecifique, Ebenostenus gen.n., avec E. iberoniger sp.n. Des clés sommaires de détermination sont données pour les espèces du genre Hirudisoma et pour les genres et espèces de Platydesmida d'Europe.

En annexe, une note nomenclaturale procède à la validation de deux taxa de rang générique. Romanosoma et Cyrnosoma, décrits respectivement par Ceuca [1967] et par Mauriès [1969] étaient restés invalides par défaut de désignation d'espèce-type. Romanosoma cavernicola Ceuca, 1967 est désigné comme espèce-type du genre Romanosoma et Neoatractosoma (Cyrnosoma) beroni Mauriès, 1969 l'est aussi pour le genre Cyrnosoma. Le nom de groupe-famille Cyrnosomatidae est par conséquent lui aussi validé.

ABSTRACT. Identification results of the epigean Platydesmida and Polyzoniida millipedes collected by
British expeditions in 1993 and 2004 in the Northwest of Iberian Peninsula (Galicia and the extreme North of Portugal) are presented. One Polyzoniida from the genus Hirudisoma and two new species of Platydesmida are described, one of which belongs to the genus Dolistenus, D. iberoalbus sp.n., the other to a new monotypic genus, Ebenostenus gen.n., with E. iberoniger sp.n. Summarizing keys are given to the species of Hirudisoma and to the genera and species of Platydesmida of Europe.

In the appended nomenclatural note, two taxa of generic rank, Romanosoma and Cyrnosoma, are validated. Described by Ceuca [1967] and Mauriès [1969], respectively, these genera remained invalid because no type-species was designated for either of them. Romanosoma cavernicola Ceuca, 1967 is selected as type-species for Romanosoma, and Neoatractosoma (Cyrnosoma) beroni Mauriès, 1969 for Cyrnosoma. The familygroup name Cyrnosomatidae is thus validated as well.

РЕЗЮМЕ. Представлены результаты определений наземных Platydesmida и Polyzoniida, собранных британскими экспедициями в 1993 и 2004 г.г. на северо-западе Иберийского полуострова (Галисия и самый север Португалии). Найден один вид Рolyzoniida из рода Hirudisoma и описаны два новых вида 
Platydesmida, один из которых принадлежит роду Dolistenus, D. iberoalbus sp.n., а другой - новый монотипический род, Ebenostenus gen.n., с видом $E$. iberoniger sp.n. Даны ключи для видов Hirudisoma и родов и видов Platydesmida фауны Европы.

В приложении валидизированы два таксона родового ранга, Romanosoma и Cyrnosoma. Описанные соответственно Ceuca [1967] и Mauriès [1969], эти рода оставались невалидными, т.к. ни для одного из них не был обозначен типовой вид. Romanosoma cavernicola Ceuca, 1967 выбран в качестве типового вида Romanosoma, a Neoatractosoma (Cyrnosoma) beroni Mauriès, 1969 — для Cyrnosoma. Название ранга семейства Cyrnosomatidae тем самым также валидизировано.

\section{Introduction}

Le présent travail est une nouvelle contribution à l'étude des diplopodes collectés par nos collègues du British Myriapod and Isopod Group en 2004, et par David Bilton en 1993. Leur efficacité, déjà soulignée [Mauriès, 2015] est confirmée par la qualité et la nouveauté du matériel récolté. Celui-ci se limite pourtant à un ensemble de diplopodes relativement petit, réunis autrefois sous le vocable de «Colobognathes », un ancien

Tableau 1. Polyzoniida et Platydesmida des missions britanniques récoltées au N.O. de la Péninsule Ibérique en 1993 et 2004. Table 1. Polyzoniida and Platydesmida collected by the British expeditions in NW of the Iberian Peninsula in 1993 and 2004. Таблица 1. Polyzoniida и Platydesmida, собранные британскими экспедициями на СЗ Иберийского полуострова в

\section{POLYZONIIDA}

Hirudisoma aff. vachoni

\begin{tabular}{|c|c|c|c|c|c|}
\hline Pays & district & loc. & coll. & date & \\
\hline \multirow{2}{*}{ PORTUGAL } & VIANA do & Castanheira, alt. $450 \mathrm{~m}$ & J.P. Richards & \multirow{2}{*}{$28-03-2004$} & $1 \mathrm{j}$. \\
\hline & CASTELO & (S.S.E. de Paredes de Coura) & Paul Lee & & $1 ?, 1 \mathrm{j}$. \\
\hline
\end{tabular}

PLATYDESMIDA

Dolistenus iberoalbus sp.n.

\begin{tabular}{|c|c|c|c|c|c|}
\hline Pays & prov. / distr. & Loc. & coll. & date & \\
\hline \multirow{12}{*}{$\begin{array}{l}\text { ESPAGNE, } \\
\text { Galicia }\end{array}$} & \multirow{8}{*}{ ORENSE } & \multirow{5}{*}{$\begin{array}{l}\text { Avion (vallée du Rio } \\
\text { Valdeiras) }\end{array}$} & R. D. Kime & \multirow{5}{*}{$26-03-2004$} & $1 \mathrm{j}$. \\
\hline & & & St. J. Gregory & & $10^{7}, 2$ + \\
\hline & & & Paul Lee & & $10^{7}$ \\
\hline & & & Helen Read & & $1 \sigma^{7}, 19,5 \mathrm{j}$. \\
\hline & & & J.P. Richards & & $1 O^{7}, 1 \sigma^{7} ?, 2$ +, , $1 \mathrm{j}$. \\
\hline & & \multirow{3}{*}{$\begin{array}{l}\text { Beade (24 km ouest de } \\
\text { Orense) }\end{array}$} & Paul Lee & \multirow{3}{*}{$26-03-2004$} & $10^{7}$ \\
\hline & & & R. D. Kime & & $10^{7}, 4$ 우, $3 \mathrm{j}$. \\
\hline & & & J.P. Richards & & $80^{7} \bigcirc^{7}, 7$ + \\
\hline & \multirow{4}{*}{ PONTEVEDRA } & \multirow{3}{*}{ Gondomar (sud de Vigo) } & J.P. Richards & \multirow{3}{*}{$24-03-2004$} & $1 \sigma^{\top}, 1 \mathrm{j}$. \\
\hline & & & R. D. Kime & & $4 O^{7} O^{7}, 2$ 우 \\
\hline & & & Paul Lee & & $10^{7}, 7$ + $\&$ j. \\
\hline & & Sud de Gondomar & St. J. Gregory & $25-03-2004$ & $3 \sigma^{x} \sigma^{x}, 1 ?$ \\
\hline \multirow{9}{*}{ PORTUGAL } & \multirow{8}{*}{$\begin{array}{l}\text { VIANA do } \\
\text { CASTELO }\end{array}$} & Senhora da Peneda & David Bilton & 06-05-1993 & $10^{7}$ j.,7??,19 \\
\hline & & \multirow{4}{*}{$\begin{array}{l}\text { Castanheira (S.S.E. de } \\
\text { Paredes de Coura) }\end{array}$} & Helen Read & \multirow{4}{*}{$28-03-2004$} & $\begin{array}{l}30 \odot^{7} \sigma^{7}, 40 \text { क् }, 7 \text { j., } 1 \\
\text { pupoïde, œufs? }\end{array}$ \\
\hline & & & St. J. Gregory & & 390 \\
\hline & & & Paul Lee & & $6 \bigcirc^{7} \sigma^{7}, 12$ 우, 3 j. \\
\hline & & & \begin{tabular}{|l} 
J.P. Richards \\
\end{tabular} & & $4 \bigcirc^{7} \bigcirc^{7}, 3$ O,+ 2 j. \\
\hline & & \multirow{3}{*}{$\begin{array}{l}\text { Vascoes (S.S.E. de Paredes } \\
\text { de Coura) }\end{array}$} & St. J. Gregory & \multirow{3}{*}{$28-03-2004$} & $5 \bigcirc^{2} \sigma^{2}, 1 \%$ \\
\hline & & & Helen Read & & $10^{7}, 390$ \\
\hline & & & Paul Lee & & $7 \bigcirc^{7} O^{7}, 5$ 우, 1 j. \\
\hline & BRAGA & $\begin{array}{l}\text { Serra do Gerês, } 10 \mathrm{~km} \text { au N. } \\
\text { de Caldas do Gerês }\end{array}$ & David Bilton & $07-05-1993$ & 22 ○ $^{7}, 6$ ○ \\
\hline \multicolumn{6}{|c|}{ Ebenostenus iberoniger gen.n., sp.n. } \\
\hline Pays & province & loc. & coll. & date & \\
\hline \multirow{5}{*}{$\begin{array}{l}\text { ESPAGNE, } \\
\text { Galicia }\end{array}$} & \multirow{5}{*}{ PONTEVEDRA } & \multirow{3}{*}{$\begin{array}{l}\text { Camposancos (extrême sud } \\
\text { de la province) }\end{array}$} & R. D. Kime & \multirow{5}{*}{ 29-03-2004 } & $10^{7}, 4$ + \\
\hline & & & \begin{tabular}{|l} 
J. Lewis \\
\end{tabular} & & $10^{7}$ \\
\hline & & & Helen Read & & $10^{7}$ \\
\hline & & \multirow{2}{*}{ Camposancos, Santa Tecla } & \multirow{2}{*}{ Paul Lee } & & \begin{tabular}{|l|}
$1 \%$ \\
1
\end{tabular} \\
\hline & & & & & $1 \mathrm{j}$. \\
\hline
\end{tabular}


sous-ordre qui a été divisé en 3 ordres [Hoffman, 1980], puis en 4 [Shelley, 2003] : Polyzoniida Gervais, 1844, Platydesmida de Saussure, 1860, Siphonocryptida Pocock, 1894 et Siphonophorida Newport, 1844. Seuls les deux premiers, qui ont des représentants en Europe, seront traités dans cette note.

Ces diplopodes, dans la zone prospectée par nos collègues, n'ont été rencontrés que dans les provinces galiciennes de Pontevedra et Orense, et dans les districts portugais de Viana do Castelo et de Braga. Jusqu'à ce jour, seul l'Atlas de Kime \& Enghoff [2011, page 146], par un point bleu sur la carte faisait état de l'existence d'un colobognathe dans cette zone, le Polyzoniida Hirudisoma vachoni Mauriès, 1964 dans le district nord-portugais de Viana do Castelo. II s'agit là certainement de l'une des nombreuses localités (restées inédites) visitées par notre ami l'infatigable prospecteur Richard Desmond Kime. Au cours de leurs missions de 1993 et de 2004, nos collègues ont bien trouvé un taxon affine de celui-ci, mais aussi et surtout ils ont découvert deux taxa nouveaux de Platydesmida, qui se rattachent, l'un au genre Dolistenus Fanzago [1875], qui était inconnu de la Péninsule Ibérique, et l'autre à un nouveau genre, Ebenostenus gen.n. Ce dernier est remarquable par sa coloration noire foncée qui s'efface après un séjour prolongé dans l'alcool. Ajoutons qu'une nouvelle localité de ce nouveau genre vient de nous être révélée par l'entremise de notre excellent collègue de l'Université d'Alcalà, José Domingo Gilgado.

Le tableau plus haut (Tableau 1) présente les stations de récolte des missions britanniques et leur contenu.

\section{Muséologie}

La plus grande partie du matériel récolté et identifié sera remise à la disposition des membres du British Myriapod and Isopod Group (BMIG), à l'exception de quelques échantillons qui seront déposés dans les collections des organismes ci-après :

CRBA : Centre de Recursos de Biodiversitat Animal, Barcelona.

MNHN : Collection Myriapodes-Onychophores du Muséum National d'Histoire Naturelle (Paris), série BA.

UAH : Collection de l'Université d'Alcalà de Henares (Madrid).

ZMUC : Musée Zoologique de l'Université de Copenhague.

ZMUM : Musée Zoologique de l'Université de Moscou.

\section{Partie Systématique}

Abréviations terminologiques utilisées dans le texte et pour les figures :

cx \& ca, cy : coxosternite des deux premières paires de pattes et ses annexes chez les 0 +

$\mathrm{k} \& \mathrm{ki}$ : hanche des gonopodes et son annexe.

P.1, P.2, P.3, etc : $1^{\text {ère }}, 2^{\text {ème }}, 3^{\text {ème }}$ paires de pattes, etc... (chiffres arabes).

pf, f, ti, ta, mt, u : les podomères télopodiaux (préfémur, fémur, tibia, tarse, métatarse, uncus).

st : stipes du gnathochilarium.
T.I, T.II, T.III, etc... : $1^{\text {er }}$ (collum), $2^{\text {ème }}, 3^{\text {ème }}$ anneau, etc... (chiffres romains).

$\mathrm{v} \& \mathrm{~s}$ : sternite et son expansion médiane.

ve, vi, op : valve externe, valve interne et opercule des vulves.

POLYZONIIDA Gervais, 1844

Anciennement membre de la cohorte des Colobognathes, l'ordre Polyzoniida, détaché de la susdite par Hoffman [1980], n'a pas vu la création d'un nouveau genre européen depuis Fanzago [1881]. Il comprenait pour Hoffman [1980] deux sous-ordres, dont l'un Siphonocryptidea Pocock, 1894 (deux genres, 7 espèces dont une caucasienne, une autre de la Macaronésie, et 5 non européennes) élevé au rang d'ordre par Shelley [2003] en a été détaché. L'ordre des Polyzoniida Gervais, 1844 sera donc seul considéré ici ; il comprend 3 familles, dont deux sont représentées en Europe : Polyzoniidae Gervais, 1844, avec le genre amphi-atlantique Polyzonium Brandt, 1837, et Hirudisomatidae Silvestri, 1896, avec la sous-famille européenne monogénérique Hirudisomatinae Silvestri, 1896, qui nous intéresse seule ici.

\section{Hirudisomatidae/inae Silvestri, 1896 \\ Genre Hirudisoma Fanzago, 1881}

Une importante contribution à la connaissance des Colobognathes de la région caucasienne, le tout récent travail de Golovatch et al. [2015] traite notamment du genre Hirudisoma. Il y est éclairci le statut d'un taxon resté mystérieux pour l'auteur, Leiosoma rosea (recte : -um) Victor, 1839, pour lequel un néotype a été désigné, dont la place dans le genre Hirudisoma est confirmée et qui se trouve être un synonyme plus ancien de Hirudisoma ponticum Lohmander, 1939. Ce genre comprend donc aujourd'hui une douzaine de taxa dont 10 en Europe, un en Algérie, et un répandu de l'Anatolie orientale à l'Azerbaïdjan :

H. pallidum (Fanzago, 1881) (= H. aprutianum Verhoeff, 1930) : Italie, de 1'Emilie à la Calabre.

H. carniolense (Verhoeff, 1899) : Slovénie et Croatie.

H. equiseti Verhoeff, 1930 : Italie, nord-est des Alpes.

H. getschmanni (Karsch, 1880) : Espagne, Asturies (Puerto de Pajares).

H. aff. getschmanni - Mauriès \& Barraqueta, 1985 : Espagne, Viscaye, Monte Gorbea.

H. hirsutum (Verhoeff, 1901) : Bulgarie, Grèce, Turquie (de chaque côté du Bosphore).

H. latum (Ribaut, 1908) : France : Pyrénées centrales et occidentales.

H. pyrenaeum (Ribaut, 1908) : France (et Espagne ?) : Pyrénées centrales et occidentales.

H. remyi Schubart, 1964 : Algérie.

$H$. roseum (Victor, 1839) $=H$. ponticum Lohmander, 1939: de l'Anatolie au Nord-ouest de l'Azerbaïdjan.

H. trivittatum Verhoeff, 1930 : France (Alpes maritimes), Italie (Piémont, Ligurie, Toscane).

H. vachoni Mauriès, 1964 : Portugal (Nord et centre). 
D'après les points bleus de l'Atlas de Kime et Enghoff [2011, pages 144 à 146], toutes les espèces seraient connues par un plus grand nombre de localités que ce que l'auteur de la présente note a pu relever dans la littérature. Par exemple, Hirudisoma pyrenaeum existerait en Espagne, sur le versant sud des Pyrénées centrales et occidentales, et $H$. vachoni ne serait pas seulement confiné au nord du Portugal, mais parviendrait vers le sud jusqu'à la région de Lisbonne. Il s'agit probablement de localités découvertes par Richard Desmond Kime, et restées jusqu'à présent inédites.

La clé des espèces du genre, publiée par Mauriès [1964] et citée par Hoffman [1980], est mise à jour, ciaprès : à noter que $H$. getschmanni qui a probablement sa place dans le genre, mais dont les caractères restent encore à fixer, n'y figure pas.

\section{Clé des espèces du GenRe Hirudisoma :}

1. Dos des métazonites ponctué ; coloration uniforme de jaunâtre à brunâtre, ou uniformément marbrée. Gonopodes antérieurs (P.9 $\left.\mathrm{O}^{\top}\right)$ à hanches pourvues sur leur face orale d'une nette apophyse digitiforme H. pallidum (= H. aprutianum).

-. Dos des métazonites plus ou moins granuleux, mais sans ponctuation nette. Hanches des gonopodes antérieurs sans prolongement digitiforme sur leur face orale (parfois une courte saillie dentiforme) ................................ 2

2. Coloration jaunâtre, avec 3 bandes longitudinales dorsales brunâtres, la médiane généralement plus foncée que les latérales, qui sont parfois presque effacées ........... 3

-. Coloration uniforme, jaunâtre ou marbrée de brun .... 5
3. Dos recouvert d'une fine pilosité ; espèces italiennes (et sud-est France) ............................................................. 4

-. Dos glabre ; espèce portugaise .................... H. vachoni

4. Sur les côtés du dos des métazonites, la strie transversale antérieure est plus prononcée que la marginale postérieure H. equiseti

-. Sur les côtés du dos des métazonites, la strie transversale antérieure est moins prononcée que la marginale postérieure ...................................... H. trivittatum

5. Bord postérieur des tergites garni d'un rang de cils assez longs et arqués. Hanches des gonopodes antérieurs (P.9 $\left.\sigma^{7}\right)$ pourvues d'un prolongement triangulaire interne érigé et d'un " peigne » de quelques fortes et courtes soies en bâtonnets

-. Bord postérieur des tergites sans cils. Hanches des gonopodes antérieurs (P.9 $\left.\mathrm{O}^{7}\right)$ sans prolongement interne érigé et sans peigne de fortes soies en bâtonnets ................. 7

6. Yeux : $2+2$ ocelles chez l'adulte ................ H. hirsutum

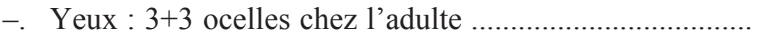
H. roseum ( $=$ H. ponticum)

7. Dos des métazonites : la strie transversale est profondément marquée sur les côtés. Article terminal des gonopodes antérieurs (P.9 $\left.\triangle^{7}\right)$ court, dépourvu de prolongement acuminé, et plutôt creusé par une petite concavité. Espèce de Croatie et Slovénie ............................. H. carniolense

-. Dos des métazonites : la strie transversale est très fine. Article terminal des gonopodes antérieurs (P.9 $\left.\sigma^{7}\right)$ pourvu d'un prolongement acuminé. Espèces pyrénéennes ... 8

8. Corps allongé, 5 à 6 fois plus long que large. Article terminal des gonopodes antérieurs (P.9 $\left.\mathrm{O}^{7}\right)$ à pointe dirigée vers l'intérieur ............................... H. pyrenaeum

-. Corps ramassé, 2,5 fois plus long que large. Article terminal des gonopodes antérieurs $\left(\mathrm{P} .9 \mathrm{O}^{7}\right)$ à pointe érigée H. latum

Tableau 2. Mensurations ( $\mathrm{mm}$ ), nombre d'anneaux (N), d'ocelles et de pattes (npp) des Hirudisoma nord-ibériques. Table 2. Measurements (mm), number of segments (N), of ocelli and of legs (npp) of northern Iberian Hirudisoma. Таблица 2. Размеры (mm), число сегментов (N), глазков и ног (npp) северно-иберийских Hirudisoma.

\begin{tabular}{|c|c|c|c|c|c|c|c|c|}
\hline & $\mathrm{N}$ & $\begin{array}{c}\begin{array}{c}\text { Longueur } \\
\text { du corps }\end{array} \\
\end{array}$ & largeur & $\mathrm{L} /$ largeur & npp. & yeux & $\begin{array}{l}\text { largeur } \\
\text { collum }\end{array}$ & $\begin{array}{l}\text { largeur du collum } \\
/ \text { largeur du corps }\end{array}$ \\
\hline \multicolumn{9}{|c|}{ Hirudisoma vachoni Mauriès } \\
\hline $\begin{array}{l}0^{7} \text { holotype } \\
\text { Peso de Regua }\end{array}$ & 21 & 3 & 1,3 & 2,30 & $26(+2)$ & $3+3$ & 1 & 0,77 \\
\hline \multicolumn{9}{|c|}{ Hirudisoma aff. vachoni - voir plus bas } \\
\hline $\begin{array}{l}q \text { Castanheira } \\
\text { coll. Paul Lee } \\
\end{array}$ & 29 & 7,3 & 1,75 & 4,17 & 48 & $3+3$ & 0,82 & 0,47 \\
\hline $\begin{array}{l}\text { juv. Castanheira } \\
\text { coll. Paul Lee }\end{array}$ & 14 & 1,8 & 0,9 & 2 & 14 & $2+2$ & 0,55 & 0,60 \\
\hline \begin{tabular}{|l|}
$q$ Castanheira \\
coll. JP.Richards
\end{tabular} & 28 & 7,1 & 1,54 & 4,60 & 46 & $3+3$ & 0,86 & 0,55 \\
\hline \begin{tabular}{|l|} 
Castanheira \\
albinos \\
\end{tabular} & 27 & 4,7 & 1,62 & 2,90 & 42 & $\begin{array}{c}3+3 \\
\text { sans pigment } \\
\end{array}$ & 0,76 & 0,47 \\
\hline \multicolumn{9}{|c|}{\begin{tabular}{|l|} 
Hirudisoma getschmanni (Karsch) \\
\end{tabular}} \\
\hline$\sigma^{7}$ Puerto Pajares & $20 ?$ & 3 & 1 & 3 & $?$ & $?$ & 0,60 & 0,60 \\
\hline \multicolumn{9}{|c|}{ Hirudisoma aff. getschmanni-Mauriès \& Barraqueta [1985] } \\
\hline$\sigma^{7}$ Viscaye & 18 & 3,2 & 1,2 & 2,6 & $\begin{array}{c}22(?) \\
+2 \\
\end{array}$ & $3+3$ & 0,74 & 0,60 \\
\hline 9 Viscaye & 16 & 2,02 & 1,16 & 1,74 & 16 & $3+3$ & 0,75 & 0,65 \\
\hline
\end{tabular}




\section{Hirudisoma aff. vachoni Mauriès, 1964}

MATERIEL. Nord du Portugal, distr. Viana do Castelo, Castanheira (4,5 km. au S.S.E. de Paredes de Coura), alt. $450 \mathrm{~m}, 28-03$ 2004, coll. Paul Lee, 1 , 1 juv., et coll. J.P. Richards, 2 $\rightarrow$ dont une albinos.

En l'absence de mâle, ce n'est qu'avec doutes que l'on peut rapporter ce matériel à $H$. vachoni. Cette identification est néanmoins plausible, la localité type de cette espèce ne se trouvant qu'à $100 \mathrm{~km}$ au sud-est de Castanheira. Et c'est ce qui explique le point bleu de l'Atlas de Kime et Enghoff [2011, page 146].

La + récoltée par Paul Lee présente un type de coloration semblable à celui du type de $H$. vachoni, mais la ligne médiodorsale de pigmentation foncée est moins nette et les latérales sont presque effacées. La strie transverse antérieure des métatergites est bien nette, non interrompue et soulignée par des microgranules de pigmentation foncée, comme l'est le bord caudal de chaque tergite. Ce dernier est très légèrement échancré de chaque côté, ce qui fait paraitre le 1/ 5 externe de chaque moitié un peu saillant vers l'arrière (ce qui n'apparaît pas sur la figure 1 de Mauriès [1964], mais ce détail a pu être vérifié car il existe chez le type). Les $3+3$ ocelles sont inscrits dans un champ pigmenté de noir. Les ozopores sont, sur les segments moyens et postérieurs, situés dans l'angle latéropostérieur de la face dorsale du métatergite, peu écartés du bord latéral, mais éloignés du bord postérieur par une distance égale au tiers de la longueur du métazonite. Le premier ozopore apparaît dès le $5^{\text {ème }}$ tergite, où, contrairement aux suivants, et seul dans ce cas, il se situe dans l'angle antérieur (émoussé) de cet anneau. Le dernier ozopore se trouve dans celui qui précède l'antépénultième, chez qui la présence d'un ozopore est douteuse.

La femelle récoltée par J.P. Richards ne se distingue pas de la précédente, sauf par la présence, au milieu des deux moitiés de presque tous les métatergites, d'une très petite dépression en ovale transverse et par la translucidité de la portion postérieure de l'angle caudal de chaque métatergite moyen.

Si ce n'était sa dépigmentation totale, y compris autour des $3+3$ ocelles, et l'absence de micro-granules pigmentés, la femelle albinos ne se distingue en rien de la première (Tableau 2).

\section{PLATYDESMIDA de Saussure, 1860}

Egalement ancien membre de la cohorte des Colobognathes, l'ordre Platydesmida se distingue des Polyzoniida par la présence de paranota (prozonites bien plus étroits que les métazonites) et l'absence d'ocelles. Il comprenait jusqu'à ce jour une quinzaine de genres et une soixantaine d'espèces [Hoffman, 1980]. Parmi les 12 genres et la trentaine d'espèces de la famille principale qui le constitue, Andrognathidae, les 8 genres et 15 espèces de la sous-famille Doliste- ninae, les 7 genres et les 12 espèces de la tribu Dolistenini, seulement 3 genres et 6 espèces sont européennes: Dolistenus Fanzago, 1874 (2 espèces), Plutodesmus Silvestri, 1903 (1 espèce) et Fioria Silvestri, 1898 (3 espèces).

A ces six espèces, s'ajoutent à présent deux nouvelles du Nord-ouest de la Péninsule Ibérique qui se rattachent, l'une au genre Dolistenus, l'autre à un nouveau genre, Ebenostenus gen.n. Elles sont incluses dans la clé ci-après :

\section{Clé sommaire des taxa européens de Platydesmida :}

1. Métazonites du tronc pourvus dorsalement de deux rangs transverses de $3+3$ (rarement $4+4$ ) tubercules. ... Fioria Silvestri, 1898 ............................................................ 2

-. Pas de tubercules en rangs transverses sur le dos des métazonites

2. Dos du collum orné de deux rangs de $2+2$ tubercules ... F. tuberculata Silvestri, 1898

Italie (Ligurie) \& France (Alpes-maritimes)*.

-. Dos du collum avec deux rangs de tubercules: un rang antérieur de $1+1$ et un rang postérieur de $2+2 \ldots \ldots \ldots . . .3$

3. Métazonites : tubercules dorsaux en granules ................. F. mediterranea (Daday, 1889), Grèce.

-. Métazonites : tubercules dorsaux allongés en cônes à pointe émoussée ........... F. demangei Mauriès, 1964, Portugal.

4. Paranota allongées, plus longues que larges, celles des premier anneaux repoussées vers l'avant ; $2^{\text {ème }}$ tergite plus large que le collum, ses paranota encadrant ce dernier ... Plutodesmus Silvestri, 1903

$$
\text { P. typhlus (Daday, 1889), Grèce. }
$$

-. Paranota pas plus longues que larges ou aussi longues que larges, celles des premiers anneaux non ou faiblement repoussées vers l'avant ................................................ 5

5. Métazonites du tronc divisés en deux zones, une antérieure et une postérieure par une dépression transversale en rigole qui court d'un bord à l'autre. Ozopores s'ouvrant à l'extrémité d'un court cylindre saillant légèrement évasé distalement. Pigmentation noire foncée sur le vivant ........... Ebenostenus iberoniger gen.n., sp.n. Espagne (Sud de la Galice)

-. Métazonites du tronc sans dépression transverse courant d'un bord à l'autre, mais avec parfois une paire de courtes dépressions allongées transversalement et situées une de chaque côté du sillon dorso-médian. Ozopores débouchant au ras des téguments. Pigmentation variant du blanchâtre au brunâtre .... g. Dolistenus Fanzago, 1874 ............ 6

6. Aucune trace de dépression transverse sur le dos des métazonites

Au dos des métazonites, une courte dépression allongé transversalement se trouve de chaque côté du sillon dorso-médian ........................ D. savii Fanzago, 1874.

Sud-Italie, Grèce, Croatie, AFN, Chypre, Turquie.

7. Moitié antérieure des prozonites parcourue par deux ou trois stries transverses circulaires D. humicolus (Verhoeff, 1926) Nord-est de l'Italie**.

-. Prozonites entièrement lisses, limités caudalement par une seule strie transverse circulaire ....... D. iberoalbus sp.n. Espagne (Galice) \& Nord-Portugal.

"Fioria hyrcana Golovatch, 1980, revu par Golovatch et al. [2015], du Sud-est de 1'Azerbaïdjan et du Nord-ouest de 1'Iran, est très proche de F. tuberculata.

${ }^{* *}$ Kime et Enghoff [2011] citent cette espèce sous le nom de D. humicola. 
Nos deux nouvelles espèces se distinguent comme suit :

\begin{tabular}{|c|c|c|}
\hline & Ebenostenus iberoniger gen.n., sp.n. & Dolistenus iberoalbus sp.n. \\
\hline Pigmentation & $\begin{array}{l}\text { Du pigment noir sur tout le corps des } \\
\text { exemplaires vivants et récemment mis en alcool. }\end{array}$ & Coloration blanchâtre à brunâtre. \\
\hline Pilosité & $\begin{array}{l}\text { Dos de la tête au telson couvert d'une pilosité } \\
\text { dense, et régulièrement disposée, à poils courts } \\
\text { et courbés. }\end{array}$ & $\begin{array}{l}\text { Dos de la tête au telson couvert d'une pilosité un } \\
\text { peu moins dense et plus anarchique, chaque poil } \\
\text { étant long et droit, rarement arqué. }\end{array}$ \\
\hline Collum & $\begin{array}{l}3 \text { fois plus large que long, et plus large que la } \\
\text { tête }(5 / 4) \text {. }\end{array}$ & $\begin{array}{l}4 \text { fois plus large que long, et de même largeur } \\
\text { que la tête. }\end{array}$ \\
\hline Premier ozopore & au T.V, en position orale. & Au T.V, en position moyenne. \\
\hline Autres ozopores & $\begin{array}{l}\text { S'ouvrant à l'embouchure d'un court cylindre } \\
\text { légèrement évasé, près du bord de l'angle caudal } \\
\text { de chaque paranota (du T.VI au prételsonien), } \\
\text { face dorsale. }\end{array}$ & $\begin{array}{l}\text { Ovale et tourné vers l'extérieur, au ras du } \\
\text { tégument, près du bord de l'angle caudal du côté } \\
\text { dorsal de chaque paranota (du T.VI au } \\
\text { prételsonien). }\end{array}$ \\
\hline Sillon dorsomédian & A partir du T.III. & A partir du T.II. \\
\hline Prozonites & $\begin{array}{l}\text { Leur partie orale est marquée de stries } \\
\text { circulaires, au nombre de } 2 \text { à } 5 \text {; leur partie } \\
\text { caudale est couverte de micro-pastilles. } \\
\end{array}$ & Pas de stries circulaires ni de micro-pastilles. \\
\hline $\begin{array}{l}\text { Entre pro- et } \\
\text { métazonite }\end{array}$ & $\begin{array}{l}\text { Pas d'étranglement entre pro- et métazonites, } \\
\text { chaque paranota paraissant aussi large } \\
\text { distalement que basalement. }\end{array}$ & $\begin{array}{l}\text { Un étranglement entre pro- et métazonites } \\
\text { détermine une échancrure de la base du bord oral } \\
\text { de chaque paranota, faisant apparaître ces } \\
\text { dernières comme plus larges distalement que } \\
\text { basalement. }\end{array}$ \\
\hline Dos des métazonites & $\begin{array}{l}\text { Une nette division transverse les divise de part } \\
\text { en part en deux zones, une antérieure et une } \\
\text { postérieure. }\end{array}$ & Pas de division transverse. \\
\hline $\begin{array}{l}\text { Gonopodes } \\
\text { antérieurs (P.9) }\end{array}$ & $\begin{array}{l}\text { Annexe interne de chaque hanche en plaque } \\
\text { rectangulaire. }\end{array}$ & $\begin{array}{l}\text { Annexe interne de chaque hanche en simple } \\
\text { prolongement triangulaire. }\end{array}$ \\
\hline $\begin{array}{l}\text { Gonopodes } \\
\text { postérieurs (P.10) }\end{array}$ & $\begin{array}{l}\text { Hanches plus larges que hautes et à bord distal } \\
\text { arrondi. } \\
\text { Métatarse à base renflée en petite sphère portant } \\
\text { un uncus en tige rectiligne qui se fractionne } \\
\text { distalement en deux branches fortement évasées } \\
\text { à leur apex. }\end{array}$ & $\begin{array}{l}\text { Hanches sub-cubiques. } \\
\text { Métatarse et uncus d'une seule pièce, à base } \\
\text { renflée s'amincissant progressivement vers un } \\
\text { apex étroit et tronqué. }\end{array}$ \\
\hline
\end{tabular}

Genre Dolistenus Fanzago, 1874

syn. : Bericostenus Verhoeff, 1926.

Suite à la révision par Minelli [1976], la diagnose de ce genre peut s'énoncer comme suit :

Corps couvert dorsalement d'une dense pilosité. Tête ogivale, à peine plus longue que large, non cachée par le collum et non encadrée par les paranota du $2^{\text {ème }}$ anneau. Prozonites pourvus ou non de stries transverses dans sa partie moyenne et parfois de micro-verrues en lunules dans sa partie postérieure. Paranota nettes, pourvues d'ozopores très latéraux qui s'ouvrent au ras du tégument. Dos des métazonites sans tubercules, pouvant ou non être affectés de dépressions transverses et/ou médiolongitudinales. Telson (anneau préanal) en ogive bien visible dorsalement et aussi longue que large.

Deux espèces sont actuellement reconnues comme appartenant à ce genre :
1) Dolistenus savii, le type du genre, connue du sud de l'Italie, de Grèce, de Croatie, de Turquie et peut-être d'Afrique du Nord [Kime et Enghoff, 2011], a pour synonyme D. menozzii Manfredi, 1933 [cf. Manfredi, 1933].

2) Dolistenus humicolus (Verhoeff, 1926), connue du nord-est de l'Italie [Verhoeff, 1926], aurait pour synonymes, selon Minelli [1976], les deux taxa décrits dans la même région par Verhoeff [1930b], dans son genre Bericostenus : fagi (Verhoeff, 1930) et ligulifer (Verhoeff, 1930).

\section{Dolistenus iberoalbus Mauriès sp.n.}

Figs 1-16.

MATERIEL. Type : Portugal, distr. Viana do Castelo, Castanheira (S.S.E. de Paredes de Coura), $\sigma^{7}$ holotype, $5 \sigma^{7} \sigma^{7}, 7$ 우 \& 7 j., 1 pupoïde, œufs, paratypes (MNHN, série BA), $10^{\top}, 1$ q paratypes (UAH), $3 \bigcirc^{7} \sigma^{7}, 3$ 우 paratypes (ZMUC), $3 \bigcirc^{7} \sigma^{7}, 3 O^{7} \sigma^{7}$ paratypes (CRBA), $18 O^{T} O^{T}, 28 O^{T} O^{T}$ paratypes (BMIG), coll. Read, le 28-03- 
2004. Même loc., même date, tous paratypes : 3 우, coll. S.J. Gregory ; $6 \bigcirc^{7} \sigma^{7}, 12$ 우, 3 j., coll. Paul Lee; $3 \sigma^{7} \sigma^{7}, 2$ 우, 2 j. coll. J.P. Richards (BMIG); $10^{7}, 1$, coll. J.P. Richards (ZMUM $\rho 2667$ )

Autres loc. : Portugal, distr. Viana do Castelo, Vascoes (S.S.E. de Paredes de Coura), le 28-03-2004, $5 \sigma^{7} \sigma^{7}, 1$, coll. S.J. Gregory ; $1 \bigcirc^{7}, 3$ 우, coll. H. Read ; $7 \bigcirc^{7} \bigcirc^{7}, 5$ 우, 1 j., coll. Paul Lee (BMIG). Portugal, distr. Viana do Castelo, Senhora da Peneda, le 06-05-1993, $7 O^{7} O^{\top}, 1+$, coll. D. Bilton (BMIG); Portugal, distr. Braga, Serra do

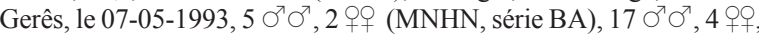
coll. D. Bilton (BMIG). Espagne, prov. Pontevedra, Gondomar, le 24 03-2004: $1 \bigcirc^{7}, 1$ j., coll. J.P. Richards ; $4 \bigcirc^{7} \sigma^{7}, 2$ + +9 , coll. R.D. Kime $; 1 \mathrm{O}^{7}, 7$ OO+, coll. Paul Lee (BMIG). Espagne, prov. Pontevedra, Sud de Gondomar, le 25-03-2004, 3 O' $\sigma^{7}, 1$ + , coll. S.J. Gregory (BMIG). Espagne, prov. Orense, Avion (vallée du Rio Valdeiras), le 26-032004 : $1 \mathrm{O}^{7}$ j., coll. R.D.Kime; $1 \mathrm{O}^{\top}, 2$ 우, coll. S.J. Gregory; $1 \mathrm{O}^{\top}$, coll. Paul Lee ; $10^{7}, 1$, 5 j., coll. H. Read ; $20^{7} \sigma^{7}, 2$, 2 , 1j., coll. J.P. Richards (BMIG). Espagne, prov. Orense, Beade (24 km à l'ouest de Orense), le 24-03-2004 : $1 \sigma^{7}$, coll. Paul Lee ; $1 \sigma^{7}, 4$ 4 , 3 j., coll. R.D. Kime ; $80^{7} \sigma^{\top}, 6$ 6 +, coll. J.P. Richards (BMIG) $; 20^{7} \sigma^{7}, 1$, , coll. J.P. Richards (MNHN, série BA).

DESCRIPTION. Coloration, longueur et nombre d'anneaux. Coloration claire, variant du blanc au brunâtre (fig. 1).

Le tableau ci-après donne le nombre d'anneaux $(\mathrm{N})$ et la longueur (L) en mm des 41 exemplaires sélectionnés parmi les plus longs et les plus petits des 236 exemplaires collectés.

Il montre que le plus grand exemplaire est une femelle de $24 \mathrm{~mm}$ de long et à 72 anneaux, le plus grand mâle ayant 57 anneaux et mesurant $16,9 \mathrm{~mm}$ de long.

Le plus petit exemplaire est un jeune ( + ?) à 19 anneaux mesurant $4,1 \mathrm{~mm}$, le plus petit mâle a 27 anneaux et mesure $5,7 \mathrm{~mm}$ de long.

Parties dorsales du corps (tête, métazonites, paranota, telson) recouvertes d'une pilosité assez dense et plutôt anarchique ; les poils sont fins et droits (fig. 3).

\begin{tabular}{|c|c|c|}
\hline Sexe & $\mathrm{L}$ & $\mathrm{N}$ \\
\hline \multicolumn{3}{|c|}{$\begin{array}{l}\text { Portugal, Viana do Castelo, Castanheira, } \\
\text { coll. H. Read }\end{array}$} \\
\hline$\sigma^{7}$ paratype & 14,7 & 54 \\
\hline$\sigma^{7}$ paratype & 14,2 & 55 \\
\hline$\sigma^{7}$ paratype & 11,6 & 46 \\
\hline$\sigma^{7}$ paratype & 11,4 & 40 \\
\hline$\sigma^{7}$ paratype & 11,0 & 46 \\
\hline$\sigma^{7}$ paratype & 10,6 & 45 \\
\hline$\sigma^{7}$ paratype & 9,3 & 37 \\
\hline$\sigma^{7}$ paratype & 8,2 & 34 \\
\hline$\sigma^{7}$ holotype & 7,8 & 32 \\
\hline$\bigcirc^{7}$ paratype & 7,3 & 32 \\
\hline o paratype & 15,0 & 58 \\
\hline o paratype & 16,7 & 57 \\
\hline o paratype & 14,6 & 56 \\
\hline O paratype & 16,5 & 53 \\
\hline o paratype & 13,4 & 49 \\
\hline o paratype & 10,7 & 42 \\
\hline q paratype & 8,7 & 34 \\
\hline o paratype & 7,8 & 34 \\
\hline o paratype & 7,0 & 31 \\
\hline \multicolumn{3}{|c|}{ Espagne, Orense, Avion, coll. R.D. Kime } \\
\hline$\sigma^{7} \mathrm{j}$. & 5,7 & 27 \\
\hline \multicolumn{3}{|c|}{ Espagne, Orense, Avion, coll. H. Read } \\
\hline j. paratype & 3,5 & 20 \\
\hline
\end{tabular}

La ligne de suture médio-dorsale est bien marquée à partir du T. II ; la dépression dans laquelle elle court est presque nulle.

Autres caractères morphologiques. Tête (fig. 2) de forme ogivale, un peu plus longue que large $(0,45 / 0,42 \mathrm{~mm})$. En arrière de chaque antenne, la capsule céphalique est marquée sur sa face ventrale par un léger étranglement qui marque ainsi deux zones : une zone orale entièrement occupée par le gnathochilarium, et une zone caudale qui est encastrée sur l'animal non disséqué sous le collum. Le gnathochilarium est un triangle plat, presque entièrement constitué par la paire de stipes pyriformes (st) qui enserrent oralement les minuscules lames linguales.

Les antennes (fig. 2) sont du type court $(0,60 \mathrm{~mm}$ de long) ; $6^{\text {ème }}$ antennomère légèrement plus long que large. Les 4 quilles sensorielles sont fusiformes.

Collum (fig. 4) légèrement plus large que la tête, 4 fois plus large que long, vaguement réniforme.

Quelques mensurations (largeurs en $\mathrm{mm}$ ) du mâle holotype : Collum (T.I): $0,44-$ T.II : 0,49 - T.III : 0,55 - T.IV : $0,57-$ T.V : 0,63 . T.VI : 0,37 au prozonite et 0,63 au métazonite - T.VIII : 0,44 au prozonite et 0,77 au métazonite. T.XX : 0,51 au prozonite et 0,78 au métazonite - T.XXVIII : 0,49 au prozonite et 0,78 au métazonite. T.XXIX : $0,74-$ T.XXX : 0,72 - T.XXXI (prételsonien) : 0,60 - Base du telson : 0,29 .

Paranota très courtes sur les anneaux antérieurs (fig. 4) et postérieurs (fig. 6), à peine plus longues sur les moyens, et là, paraissant, en vue dorsale, légèrement plus longues distalement que basalement, malgré l'effacement de leur angle oral, en raison de la présence, entre prozonite et métazonite, d'une nette échancrure (flèche de la figure 8). La pilosité de leur face dorsale est remplacée face ventrale par un réseau serré de

\begin{tabular}{|c|c|c|}
\hline Sexe & $\mathrm{L}$ & $\mathrm{N}$ \\
\hline \multicolumn{3}{|c|}{$\begin{array}{c}\text { Portugal, Braga, Serra do Gerês, } \\
\text { coll. D. Bilton }\end{array}$} \\
\hline$\sigma^{7}$ & 13,6 & 54 \\
\hline$\sigma^{x}$ & 13,2 & 51 \\
\hline$\sigma^{7}$ & 10,4 & 45 \\
\hline$\sigma^{7}$ & 8,8 & 40 \\
\hline$\sigma^{\pi}$ & 8,2 & 37 \\
\hline q & 19,2 & 65 \\
\hline q & 7,4 & 35 \\
\hline \multicolumn{3}{|c|}{ Espagne, Orense, Beade, coll. J.P. Richards } \\
\hline$\sigma^{x}$ & 16,9 & 57 \\
\hline$\sigma^{7}$ & 16,0 & 62 \\
\hline$\sigma^{\top}$ & 14,1 & 51 \\
\hline$\sigma^{7}$ & 13,2 & 54 \\
\hline$\sigma^{7}$ & 13,2 & 49 \\
\hline 9 & 24,0 & 72 \\
\hline q & 19,8 & 67 \\
\hline q & 18,0 & 61 \\
\hline q & 13,5 & 52 \\
\hline q & 8,1 & 30 \\
\hline q & 7,6 & 31 \\
\hline q (j. ?) & 5,3 & 21 \\
\hline \multicolumn{3}{|c|}{ Portugal, Viana do C., Vascoes, coll. P. Lee } \\
\hline j. & 4,1 & 19 \\
\hline
\end{tabular}




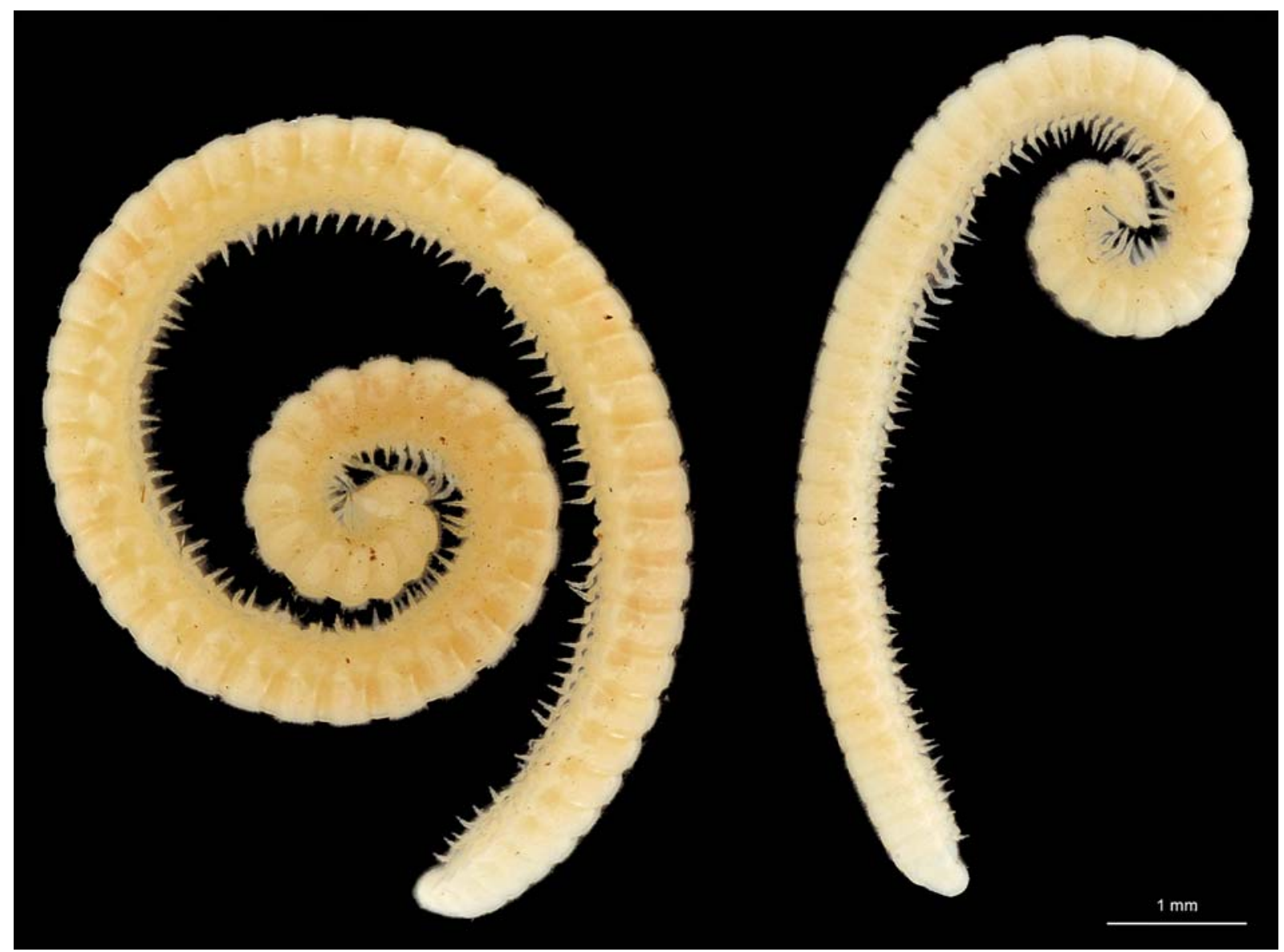

Fig. 1. Habitus de Dolistenus iberoalbus sp.n., de droite à gauche, $\sigma^{7}$ et $\odot$ paratypes du ZMUM, vue latérale. Photographie par J. Brecko. Fig. 1. Habitus of Dolistenus iberoalbus sp.n., from right to left, $O^{7}$ and $q$ paratypes from ZMUM, lateral view. Photograph by J. Brecko. Рис. 1. Общий вид Dolistenus iberoalbus sp.n., справа налево, паратипы О7 и + из ZMUM, сбоку. Фотография J. Brecko.

micro-pastilles. Les ozopores, qui apparaissent au T.V en position marginale moyenne, se trouvent en position marginale caudale sur les autres anneaux, du T.VI au prételsonien (fig. 6); ils s'ouvrent au ras du tégument, leur ouverture orientée vers l'extérieur (fig. 8). Le dos des métazonites ne présente aucune dépression transverse.

Les prozonites, contrairement à la plupart des espèces de la tribu, ne présentent aucune strie circulaire, à l'exception de celle qui limite postérieurement le prozonite. La marge antérieure de ce dernier est fortement rebordée.

L'anneau telsonien (fig. 6) ne présente aucune particularité notable.

Les pattes sont courtes (en moyenne un peu plus longues que la moitié de la largeur d'un anneau moyen), composées de 6 podomères (même aux P.1 et P.2) et d'un uncus simple ; à partir des P.3, leur sternite est orné oralement d'une puissante expansion médiane érigée digitiforme (s) et, sur presque toutes les pattes (sauf plusieurs paires de prételsoniennes), les hanches présentent des orifices de sacs coxaux (a, fig. 3). Quant aux métatarses, ils sont prolongés par un uncus simple, accompagné à sa base par une minuscule dent ; un peigne métatarsal existe aus P.1 (7 dents), aux P.2 ( 9 dents) et même aux P.3 (10 dents).

Caractères sexuels des mâles. Il ne semble pas y avoir de nettes différences de taille entre les deux sexes, les mâles paraissant généralement plus petits. On peut néanmoins les distinguer facilement par un examen latéral à un faible grossissement car ils montrent, au niveau du T.VII, une expansion

Fig. 2-6. Dolistenus iberoalbus sp.n., $\bigcirc^{7}$ holotype et $\sigma^{7}$ paratype : 2 — tête et antenne droite de l'holotype, face ventrale; 3 — vue ventrale du côté droit des $7^{\text {'̇me }}$ et $8^{\text {ème }}$ anneaux d'un $0^{7}$ paratype, montrant l'emplacement de l'expansion pleurale du $7^{\text {'me }}$, des P. 8, des gonopodes (P.9 $\&$ P.10) et des P.11 en place ; 4 - moitié gauche des 5 premiers anneaux du même $\sigma^{7} ; 5-6^{\text {ème }}$ anneau du même $\sigma^{7}$, face caudale ; $6-$ derniers anneaux du même $O^{7}$, face ventrale.

Figs 2-6. Dolistenus iberoalbus sp.n., $\sigma^{7}$ holotype and $\sigma^{7}$ paratype: 2 - head and right antenna of holotype, ventral view; 3 - ventral view of right side of segments 7 and 8 of a $0^{7}$ paratype, showing the place of pleural expansion of $7^{\text {th }}$ segment, legs 8 , gonopods (legs 9 and 10 ) and legs 11 in situ; 4 - left half of first five segments in the same $\sigma^{7} ; 5$ - segment 6 of the same $\sigma^{7}$, caudal view; 6 - last segments of the same $0^{7}$, ventral view.

Рис. 2-6. Dolistenus iberoalbus sp.n., голотип О и паратип О $: 2$ — голова и правая антенна голотипа, снизу; 3 - вид снизу правой стороны сегментов 7 и 8 паратипа $0^{7}$, показывающий положение плейрального расширения 7-го сегмента, ноги 8, гоноподы (ноги 9 и 10) и ноги 11 на месте; 4 - левая половина первых пяти сегментов того же Оج; 5 - сегмент 6 того же Оㄱ, сзади; 6 - последние сегменты того же $\bigcirc^{7}$, снизу. 

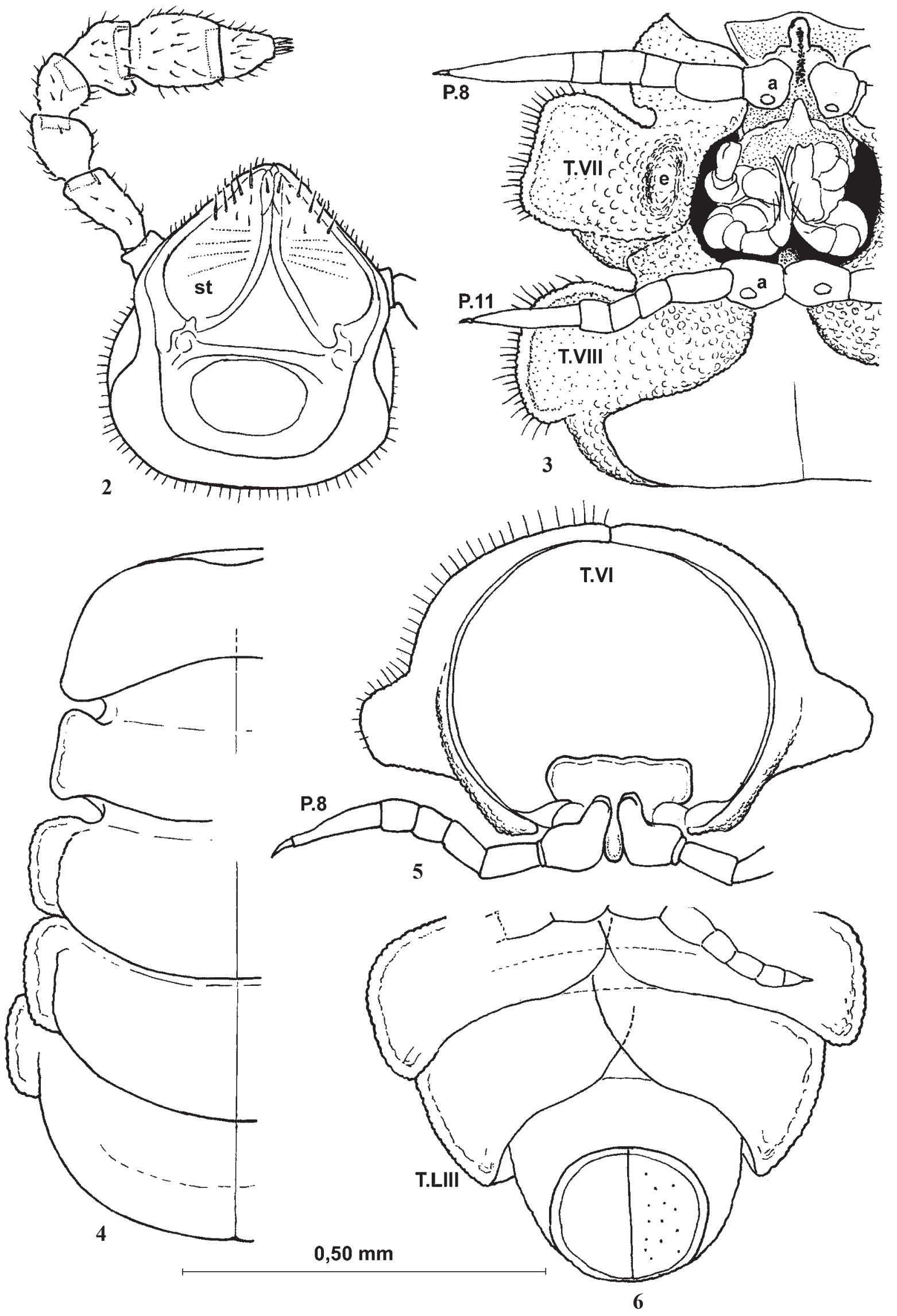
pleurale (e, fig. 3) qui paraît plus ou moins développée selon les individus. Les figures $3 \& 7$ montrent deux vues ventrales des anneaux T.VII \& T.VIII qui permettent de voir l'emplacement des P.8, des gonopodes (P.9 et P.10) et des P.11. Les gonopodes ne se distinguent de ceux des autres espèces du genre que par d'infimes détails.

Gonopodes antérieurs (P.9) (figs 9-10) : Ils ressemblent à ceux de D. humicolus, alias ligulifer Verhoeff, 1930, mais s'en distinguent néanmoins comme suit :

$\mathrm{Au}$ sternite, le prolongement médian (s) est plus robuste. Les hanches $(\mathrm{k})$ sont plus courtes. Au télopodite, le préfémur (p) est court et dépourvu de prolongement parasagittal. Le fémur (f) et le tarse (ta), distincts face orale, sont indistincts face caudale et forment une imposante masse au coude de l'organe ; quant au métatarse (mt), il se prolonge en une lame vaguement bilobée qui rappelle la ligule observée par Verhoeff [1930b].

Gonopodes postérieurs (P.10) (figs 11-13) : Construction générale comme chez tous les platydesmides, ressemblant beaucoup à la figure 24 de $D$. humicolus de Verhoeff [1930b], avec apophyse sternale médiane (s) discrète, hanches $(\mathrm{k})$ sub-cubiques et télopodites à deux courbures : une externe entre préfémur (pf) et fémur (f), et une autre interne, presque sagittale, à la base du métatarse $(\mathrm{mt})$. Ce dernier article, résultat de la fusion métatarse + uncus, est le seul qui possède des caractéristiques spécifiques ; sa base renflée émet un long prolongement qui s'amincit progressivement jusqu'à un apex étroit et tronqué. Sur sa face orale, ce podomère semble se fissurer en deux pseudoflagelles (q) et (r) tandis que sa face ventrale peut porter de une, chez le mâle holotype (fig. 13) à trois fortes soies arquées, comme chez un grand mâle paratype à 54 anneaux (figs 11-12).

Caractères sexuels des femelles. Comme chez les mâles, les P.1 et P. 2 ont 6 podomères ; le terminal (métatarse) outre sa pilosité banale, est orné sur sa face orale d'un «peigne » d'une dizaine de dents (figs 15-16). Le podomère basal, le plus large, vaguement triangulaire, la hanche, peut être considéré comme un coxosternite (cx, fig. 14), le sternite ne paraissant pas exister en tant que tel. Et si les coxosternites des P.1 ne présentent aucune particularité, il n'en est pas de même de ceux des P.2. Ces derniers sont en effet, chacun sur sa face caudale, creusés d'une grande cavité (ca) dans laquelle se loge la vulve (fig. 14). Celle-ci, de forme vaguement globuleuse, présente les parties caractéristiques des vulves de diplopodes : opercule (op) en face orale, valves (ve \& vi) en face caudale. A la base de la gouttière apodématique (entre les deux valves), on voit par transparence une paire de petits diverticules hémisphériques (réceptacles séminaux ?).

\section{Ebenostenus Mauriès gen.n.}

DIAGNOSE : Remarquable par la présence d'une nette rigole transversale qui partage en deux zones, une antérieure et une postérieure, le dos des métazonites. Ozopores débouchant à l'apex d'un court tube cylindrique saillant à l'extrémité des paranota. Présence d'une pigmentation noire foncée sur le vivant. Dos, de la tête au telson, couvert d'une pilosité très dense, très régulièrement disposée, faite de soies courtes et arquées.

ETYMOLOGIE : de la couleur de l'ébène ( $\varepsilon \beta \varepsilon v o \varsigma)$.

ESPĖCE-TYPE : Ebenostenus iberoniger sp.n., monotypie et désignation présente.

\section{Ebenostenus iberoniger Mauriès sp.n. \\ Figs 17-32.}

MATERIEL . Loc. type : Espagne (Galice), prov. Pontevedra, Camposancos, 29-03-2004, $0^{7}$ holotype, o paratype ; coll. R.D. Kime (MNHN, série BA) ; 1 t paratype, coll. R.D. Kime (ZMUM p2668) ; 2 ㅇ paratypes, coll. R.D. Kime (BMIG); $1 \mathrm{O}^{7}$ paratype coll. J. Lewis, (BMIG) ; $10^{7}$ paratype, coll. Helen Read (MNHN, série $\mathrm{BA}$ ).

Autres loc. : Espagne (Galice), prov. Pontevedra, Camposancos, Santa Tecla, 29-03-2004, 1 o, 1j., coll. Paul Lee (BMIG). Espagne (Galice), prov. Pontevedra, O Grove, Mirador Siradella, 21-03-2015, 1 + (UAH), 1 q (MNHN, série BA), coll. E. Ledesma, leg. J.D. Gilgado.

\section{Mensurations :}

\begin{tabular}{|c|c|c|c|c|c|}
\hline Loc. & Collecteur & sexe & $\begin{array}{c}\mathrm{L}, \\
\mathrm{mm}\end{array}$ & $\mathrm{N}$ & $\begin{array}{c}\text { Paires de } \\
\text { pattes }\end{array}$ \\
\hline \multirow{7}{*}{$\begin{array}{l}\text { Campo- } \\
\text { sancos }\end{array}$} & \multirow{5}{*}{ R.D. Kime } & $\sigma^{7} \mathrm{HT}$ & 15 & 50 & 92 \\
\hline & & P PT & 17,2 & 58 & 109 \\
\hline & & 9 PT & 15,4 & 58 & 107 \\
\hline & & $9 \mathrm{PT}$ & 16,6 & 56 & 103 \\
\hline & & P PT & 11,6 & 44 & 77 \\
\hline & \begin{tabular}{|l|} 
J. Lewis \\
\end{tabular} & $\sigma^{7} \mathrm{PT}$ & 14,4 & 52 & 94 \\
\hline & H. Read & $\sigma^{7}$ PT & 11,6 & 46 & 81 \\
\hline \multirow{2}{*}{$\begin{array}{l}\text { Santa } \\
\text { Tecla }\end{array}$} & \multirow{2}{*}{ Paul Lee } & 9 & 18,2 & 56 & 104 \\
\hline & & j. & 4,5 & 23 & 36 \\
\hline \multirow{2}{*}{ O Grove } & \multirow{2}{*}{ E. Ledesma } & 9 & 15,7 & 57 & \\
\hline & & 9 & 15,3 & 52 & \\
\hline
\end{tabular}

HT — holotype, PT — paratype

Fig. 7-16. Dolistenus iberoalbus sp.n., $\bigcirc^{7}$ holotype, $\odot^{7}$ et 9 paratypes : 7 - les gonopodes et paragonopodes d'un $\odot^{7}$ paratype en place, entre P.8 et P.11; 8 - paranota gauche du $26^{\text {ème }}$ anneau du $\sigma^{7}$ holotype (la flèche indique l'échancrure entre pro- et métazonite) ; 9 - gonopode (P.9) du même, face orale, vue de biais ; 10 - gonopodes (P.9) d'un O' paratype, face orale ; 11 — paragonopodes (P.10) du même, face orale ; 12 - podomère distal d'un paragonopode du même, face orale ; 13 — face ventrale du paragonopode droit du $O^{T}$ holotype ; 14 - face caudale de la base d'une P. 2 et de la vulve du côté gauche d'une + paratype ; 15 — métatarse d'une P.2 de la même $+; 16$ — métatarse d'une P.1 de la même + . Les 3 échelles : 0,20 mm.

Fig. 7-16. Dolistenus iberoalbus sp.n., $O^{7}$ holotype, $\sigma^{7}$ and $\odot$ paratypes: 7 - gonopods and paragonopods of a $\bigcirc^{7}$ paratype in situ, between legs 8 and 11;8 - left paranotum of segment 26 of $\sigma^{7}$ holotype (arrow showing the stricture between pro- and metazonite); 9 - gonopod (leg 9) of the same $\sigma^{7}$, anterior skewed view; 10 — gonopods (leg 9) of a $\odot^{7}$ paratype, anterior view; 11 — paragonopods (legs 10) of the same $\sigma^{7}$, anterior view; 12 - distal podomere of a paragonopod of the same $\sigma^{7}$, anterior view; 13 - ventral view of right paragonopod of $O^{7}$ holotype; 14 - posterior view of base of leg 2 and of left vulva in 9 paratype; 15 - metatarsus of leg 2 of the same $+; 16$ - metatarsus of leg 1 of the same + . Three scale bars: $0.20 \mathrm{~mm}$.

Рис. 7-16. Dolistenus iberoalbus sp.n., голотип О7, паратип О7 и $9: 7$ - гоноподы и парагоноподы паратипа на месте, между ногами 8 и 11; 8 - левый паратергит сегмента 26 голотипа Оㄱ (стрелка показывает сужение, разделяющее про- и метазонит); 9 — гонопод (нога

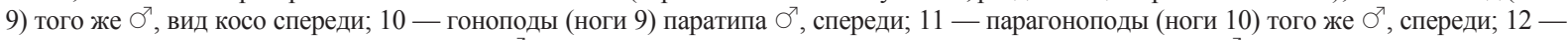
дистальный членик парагонопода того же $0^{7}$, спереди; 13 - вид снизу правого парагонопода голотипа $0^{7} ; 14-$ вид сзади основания ноги 2 и левой вульвы у паратипа $+; 15$ - метатарсус 2 той же $+; 16$ - метатарсус 1 той же + . Три масштаба: 0,20 мм. 

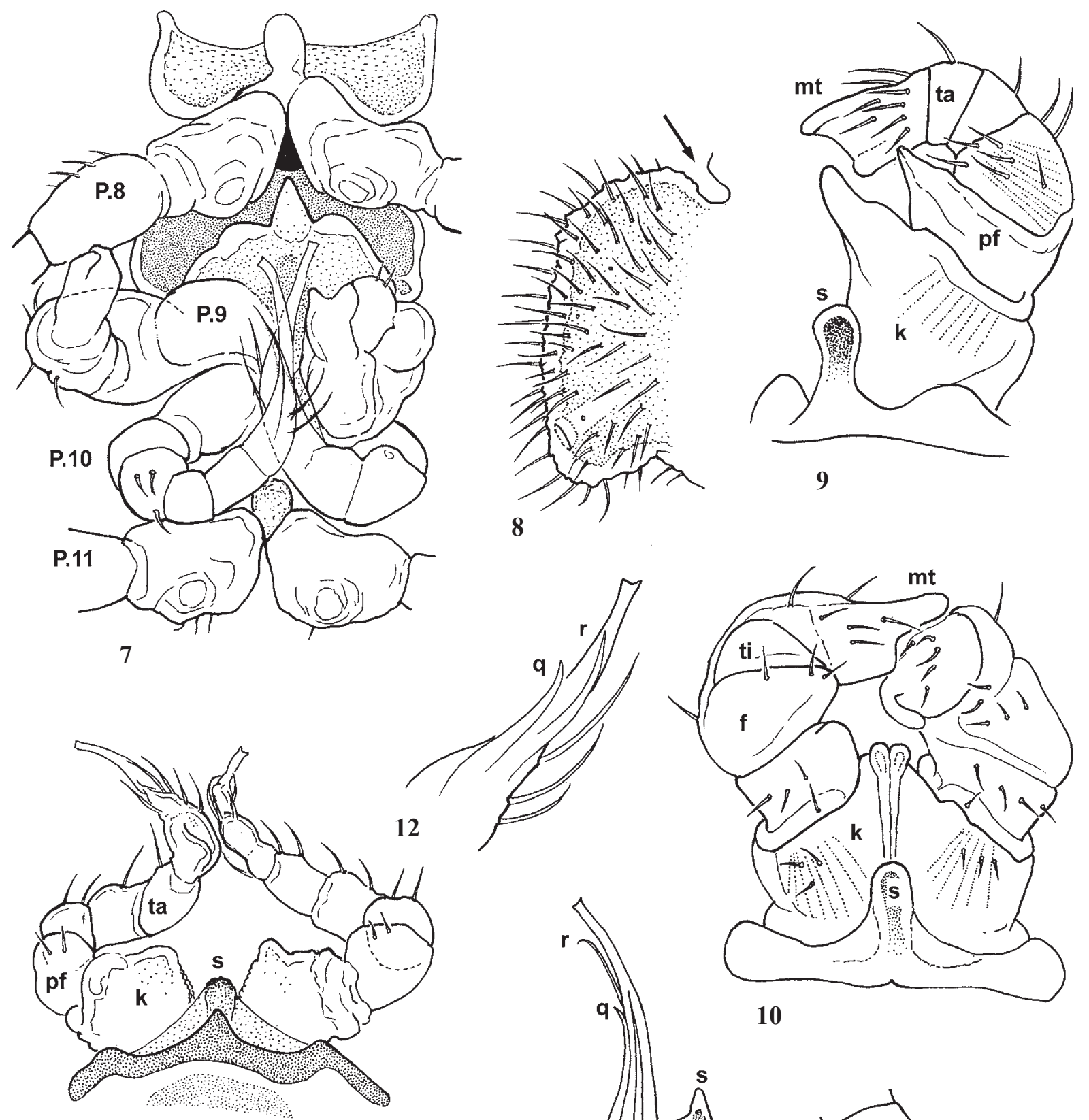

11

15
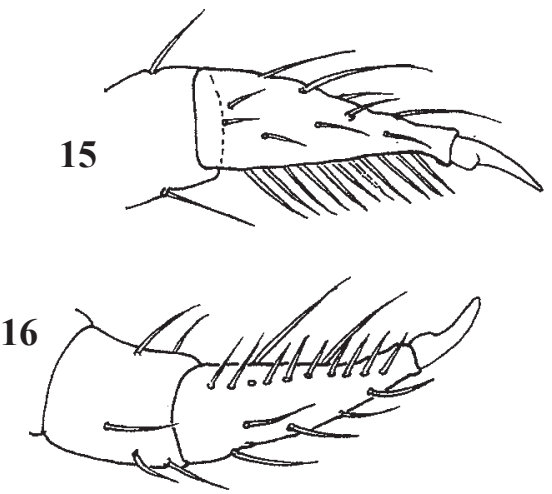

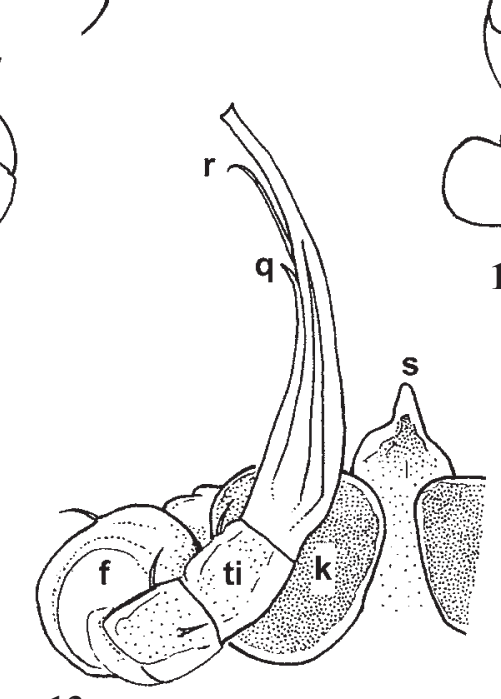

13

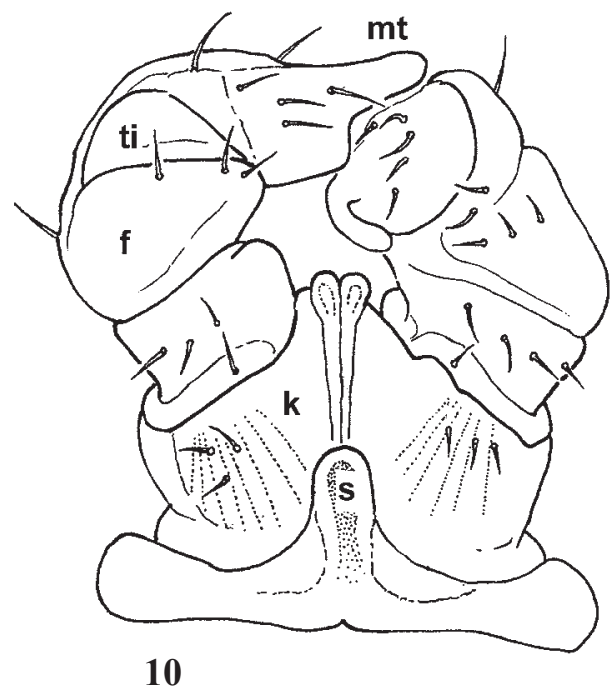

10

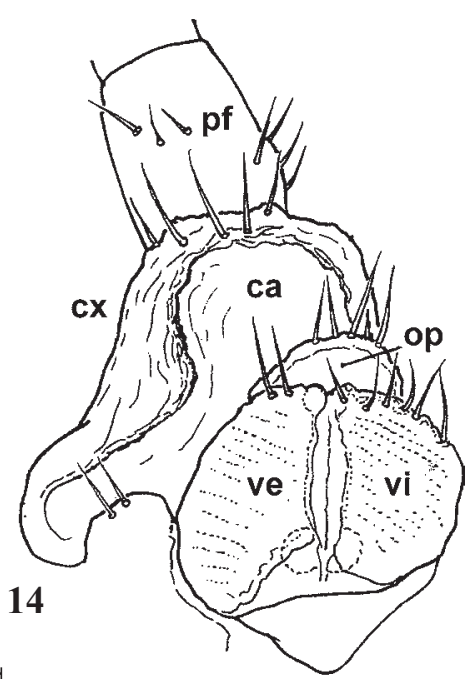

$9,10,12-16$ 

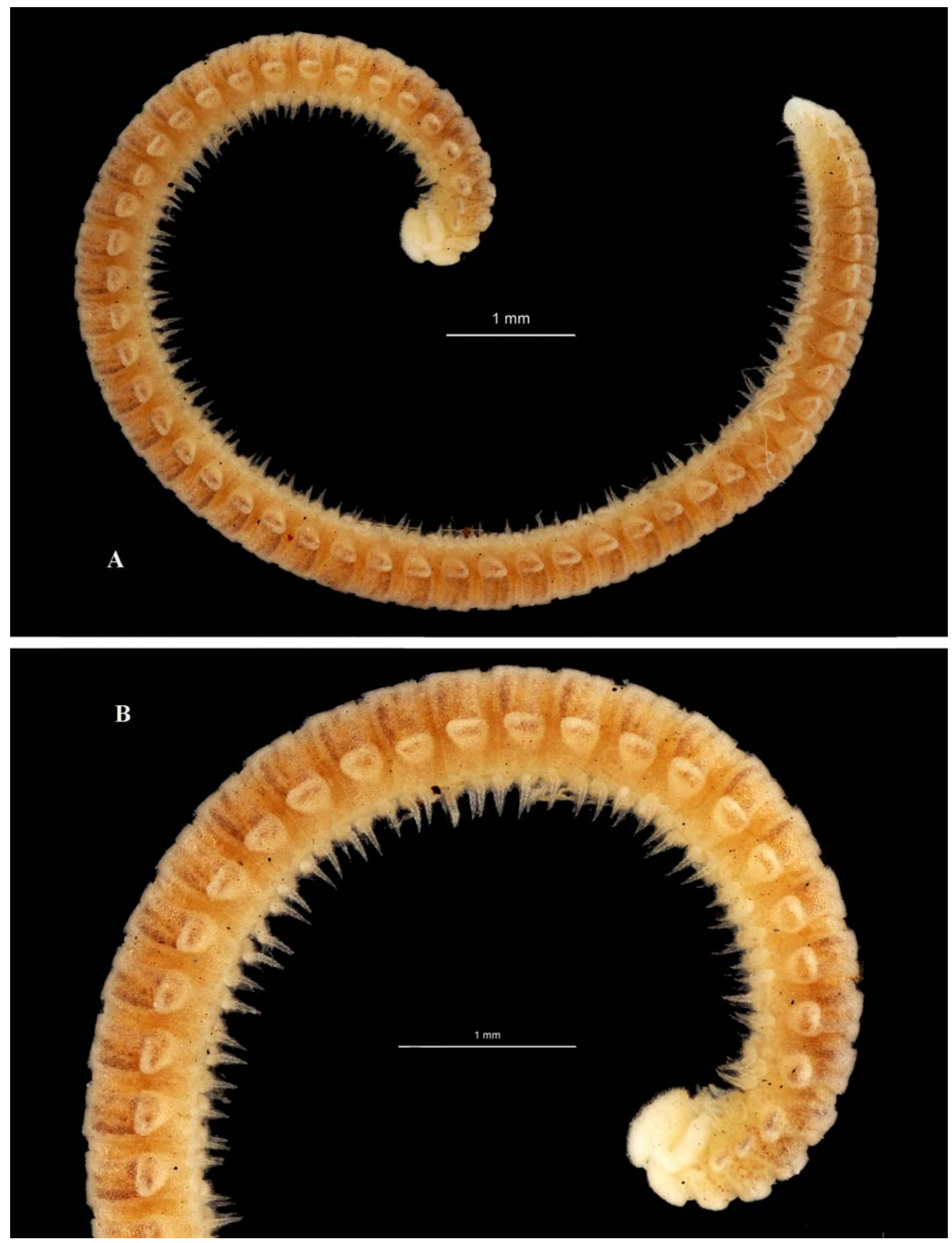

Fig. 17. Ebenostenus iberoniger gen.n., sp.n., + paratype du ZMUM. A — habitus, vue latérale ; B — partie antérieure de la même, vue latérale. Photographie par J. Brecko.

Fig. 17. Ebenostenus iberoniger gen.n., sp.n.,,+ paratype from ZMUM. A — habitus, lateral view; B — anterior part of the same $q$, lateral view. Photographs by J. Brecko.

Рис. 17. Общий вид Ebenostenus iberoniger gen.n., sp.n., паратип + из ЗМУМ. А - общий вид, сбоку; В — передняя часть тела этой же 
DESCRIPTION. Caractères morphologiques externes du mâle holotype. 50 anneaux (telson compris) et 92 paires de pattes. Longueur totale : $15 \mathrm{~mm}$. Largeurs $(\mathrm{mm})$ : du collum : 0,50 ; du T.II : 0,64 ; du T.III : 0,75 ; du T.IV : 0,76 ; du T.V : 0,78 ; du T. VI ; 0,86. Largeurs (prozonite/métazonite, $\mathrm{mm}$ ) : du T.VII : 0,45/0,81; du T.VIII : 0,47/0,82; du T. XXV : 0,48/ $0,72 ;$ du T.IIL : $0,53 / 0,73 ;$ du T.IL : $0,46 / 0,58)$.

Parties dorsales du corps (tête, métazonites, paranota, telson) entièrement recouvertes d'une dense et fine pilosité de soies courtes et courbes, espacées avec une remarquable régularité (figs 17, 24-25). Une pigmentation d'un noir profond, en flocons, ne subsiste que sur les parties du corps conservées en préparation microscopique dans la glycérine ; cette pigmentation, dont il existait encore des traces nombreuses en 2010, a disparu en 2015 dans toutes les parties conservées dans l'alcool (fig. 17). Il n'en reste que des traces sur la partie caudale des métazonites et sur les paranota de quelques individus.

La ligne de suture médio-dorsale, qui souligne la légère dépression médio-dorsale des métazonites, est bien marquée à partir du T.III et jusqu'à l'anneau prételsonien.

C'est à partir du T.IV que l'on peut observer par un simple examen latéral de l'habitus de chaque spécimen, la division des métazonites en deux zones, une antérieure et une postérieure (voir plus loin).

Tête (fig. 18) de forme ogivale, sans particularité notable, mesurant $0,4 \mathrm{~mm}$ de long sur 0,3 de large. Gnathochilarium du type court, en demi-cercle, comme chez D. humicolus, et sans traces très nettes de divisions; on distingue néanmoins assez bien la paire de stipes (st) et les petites lames linguales (fig. 19), qui sont plus nettes que chez $D$. iberoalbus sp.n.

Antennes (fig. 18) sans originalité : le $6^{\text {ème }}$ antennomère, non claviforme, est 1,4 fois plus long que large. Les 4 quilles sensorielles apicales sont en forme de bouteille allongée.

Collum (fig. 21) apparaissant en ovale transverse, 3 fois plus large que long, en vue dorsale. Sur sa face ventrale, il est très largement échancré pour abriter et cacher la moitié postérieure de la capsule céphalique.

Paranota approximativement aussi longues que larges, aux angles si émoussés, que leur contour est presque arrondi ; les plus antérieures ne sont que légèrement repoussées vers l'avant (fig. 21) et les plus postérieures, notamment la dernière paire, qui est plus petite que les précédentes, le sont vers l'arrière (fig. 23).

Prozonites glabres : à celui du T.VII, on observe deux zones ; la zone postérieure, qui occupe environ un tiers de la longueur du prozonite, est ornée de nombreuses micro-pustules rondes légèrement convexes (figs 23-24) : les $2 / 3$ antérieurs sont parcourus par 3 stries transverses qui s'incurvent vers l'arrière au contact du sillon dorso-médian, et dont seules les deux premières se prolongent sur les flancs. Les prozonites des autres anneaux sont ornés de la même manière, sauf que le nombre de stries transverses, qui atteint 5 sur le T.IX, a ensuite tendance à diminuer progressivement, à tel point qu'au $48^{\text {ème }}$ (T.IIL), il n'en subsiste plus qu'une seule.

Chaque paranota est pourvue, à partir du T.V, d'un ozopore qui s'ouvre très latéralement au bout d'un court cylindre très légèrement évasé distalement (fig. 25). Situé en position antérieure au T.V, en position moyenne au T.VI, il est post-latéral sur tous les autres anneaux, jusqu'au prételsonien (fig. 23).

Chaque métazonite (à partir du T.IV) se signale, outre la faible dépression longitudinale dans laquelle court le sillon dorso-médian (fig. 23) par l'existence d'une étroite et nette dépression transverse presque glabre et tapissée de micropastilles, qui n'existe pas chez les autres espèces de la tribu et qui affecte toute la largeur du métazonite en le partageant en deux zones pourvues de pilosité : une antérieure et une postérieure, celle-ci étant un peu plus importante que l'antérieure. La figure 24 montre la moitié gauche d'un anneau moyen (T.XXV) incliné sur le côté pour bien mettre en évidence cette division, qui est en partie dissimulée par la pilosité ; elle est plus marquée dans la moitié postérieure du corps.

Telson en ogive mesurant $0,23 \mathrm{~mm}$ de long sur 0,29 de large.

Pattes ambulatoires : toutes les pattes, même les P.1 et P.2, qui sont un peu plus courtes que les autres, se composent de 6 podomères et d'un uncus grêle, le tout mesurant chez une patte de la région moyenne du corps autour de $0,10,0,01,0,11,0,05$, $0,07,0,06,0,15$ et 0,03 , pour un total de $0,58 \mathrm{~mm}$. A partir des P.3, chaque sternite est précédé, comme chez D. iberoalbus sp.n., d'un prolongement impair érigé fusiforme (s), court sur les premières paires et devenant plus grand sur les autres et surtout aux postérieures. La paire d'hémipénis est très discrète sur la face caudale des hanches des P.2.

Caractères sexuels des mâles. Gonopodes antérieurs (figs 26-27) assez différents de ceux des autres espèces. En effet, si le sternite (v), simple bandeau oral surmonté médialement d'un prolongement érigé (s), n'est pas très différent de celui de D. humicolus, il n'en est pas de même des autres pièces :

- Les télopodites sont courts, comme chez les autres espèces, mais leurs podomères, sauf le plus basal, le préfémur présumé (pf), et peut-être aussi le fémur (f), sont indistincts ; les podomères distaux ne formant qu'une seule masse $(\mathrm{tt})$ qui s'orne d'une vague languette externe (le) qui rappelle, comme chez D. iberoalbus sp.n., celle observée et dessinée par Verhoeff [1930b, Abb. 23 \& 27] chez D. humicolus.

- Les hanches $(\mathrm{k})$ présentent une structure qui singularise l'espèce ; chacune émettant vers l'intérieur une imposante

Fig. 18-25. Ebenostenus iberoniger gen.n., sp.n., $0^{7}$ holotype : 18 - face ventrale de la tête avec l'antenne gauche ; 19 - les lames linguales, grossies (x 10) ; 20 — schéma de la face caudale du $8^{\text {ème }}$ anneau isolé, avec paragonopodes et base des P. $11 ; 21$ — schéma de la face dorsale des 6 premiers anneaux ; 22 - schéma de la face ventrale du $7^{\text {ème }}$ anneau (les gonopodes en place) ; 23 — face dorsale du telson et des deux derniers anneaux anté-telsoniens ; 24 - face dorsale de la moitié gauche du $25^{\text {ème }}$ anneau, vue de biais pour mettre en évidence la dépression transverse du métazonite ; 25 - cylindre ozoporifère d'un $0^{7}$ paratype.

Fig. 18-25. Ebenostenus iberoniger gen.n., sp.n., $\bigcirc^{7}$ holotype: 18 - ventral view of head with left antenna, with 19 - lamellae linguales, enlarged (x 10); 20 - schematic presentation of caudal face of an isolated segment 8 , with paragonopods and base of leg $11 ; 21$ - schematic presentation of dorsal face of first six segments; 22 — schematic presentation of ventral face of segment 7 (gonopods in situ); 23 - dorsal face of telson and last two pre-telson segments; 24 - dorsal face of left half of segment 25 , view skewed for showing a transverse depression on metazonite; 25 - ozoporiferous cylinder in $0^{7}$ paratype.

Рис. 18-25. Ebenostenus iberoniger gen.n., sp.n., голотип О7: 18 - вид снизу головы и левой антенны, с 19 - lamellae linguales, увеличено (х 10); 20 - схема задней стороны изолированного сегмента 8 с парагоноподами и основанием ноги $11 ; 21$ - схема спинной стороны первых шести сегментов; 22 - схема нижней стороны сегмента 7 (гоноподы на месте); 23 - спинная сторона тельсона и двух последних сегментов перед ним; 24 - спинная сторона левой половины сегмента 25, вид скошен, чтобы показать поперечное углубление на метазоните; 25 - несущий пору цилиндр у паратипа $\mathrm{O}^{7}$. 

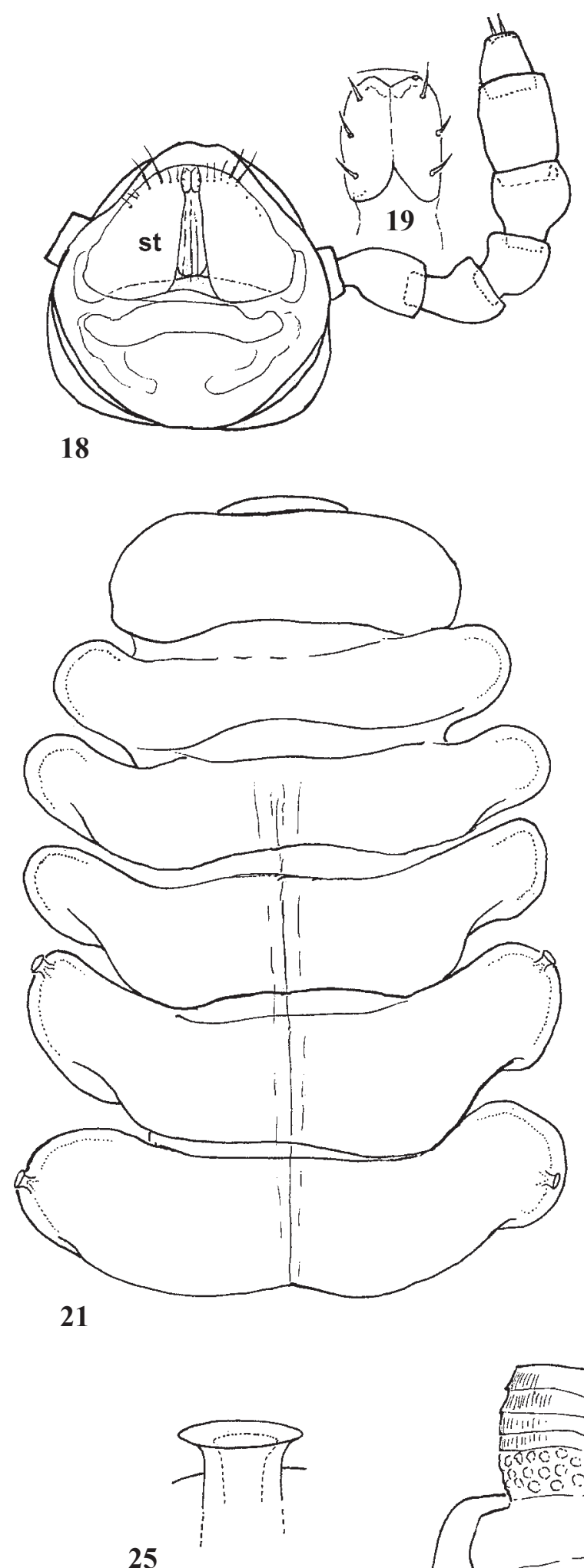

$\frac{25}{0,05 \mathrm{~mm}}$

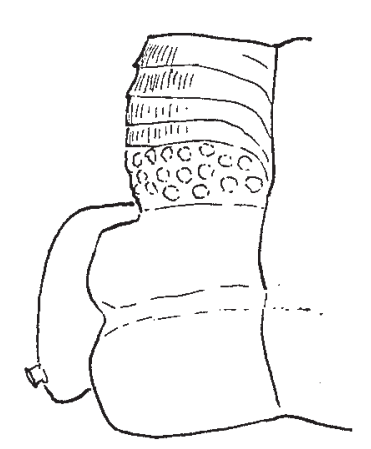

24
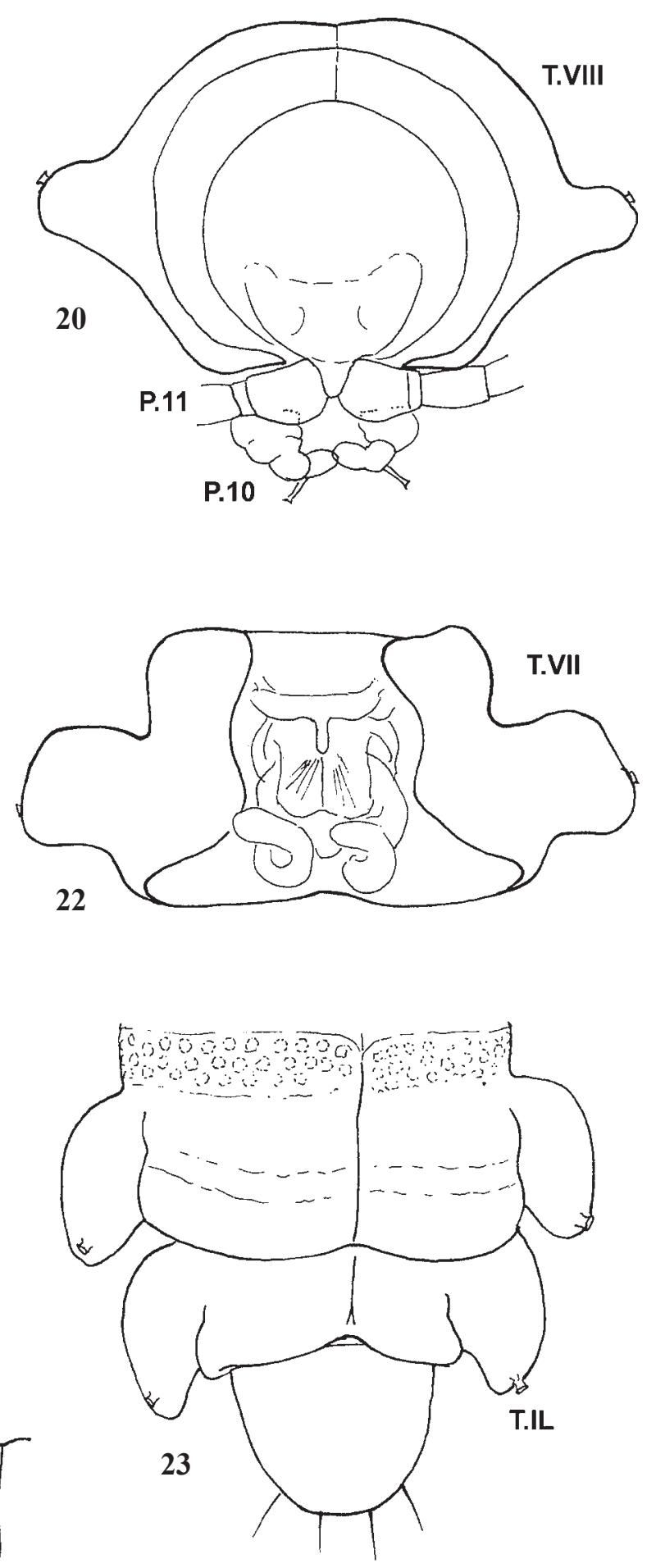

18-24

$0,50 \mathrm{~mm}$ 


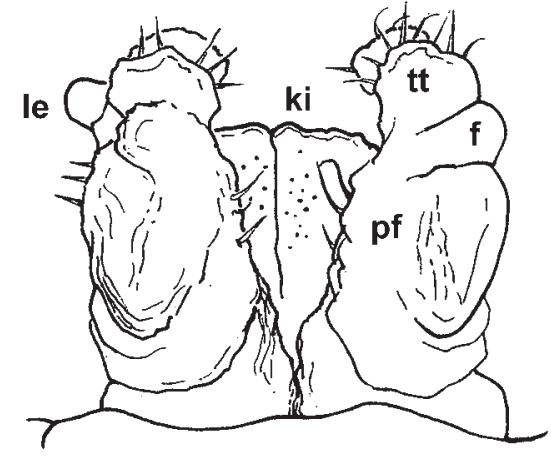

26

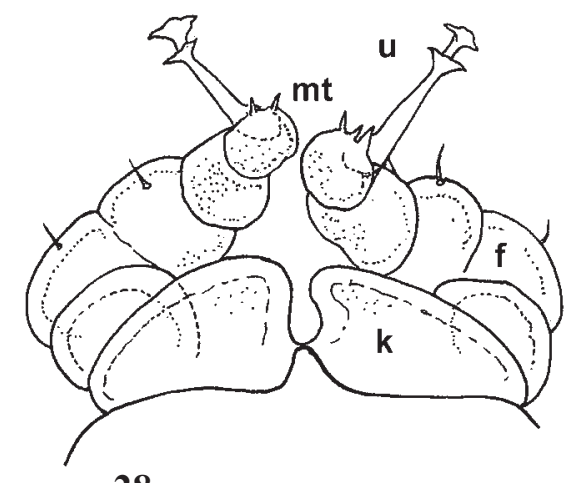

28

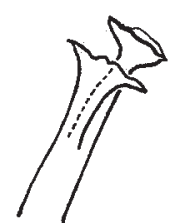

29
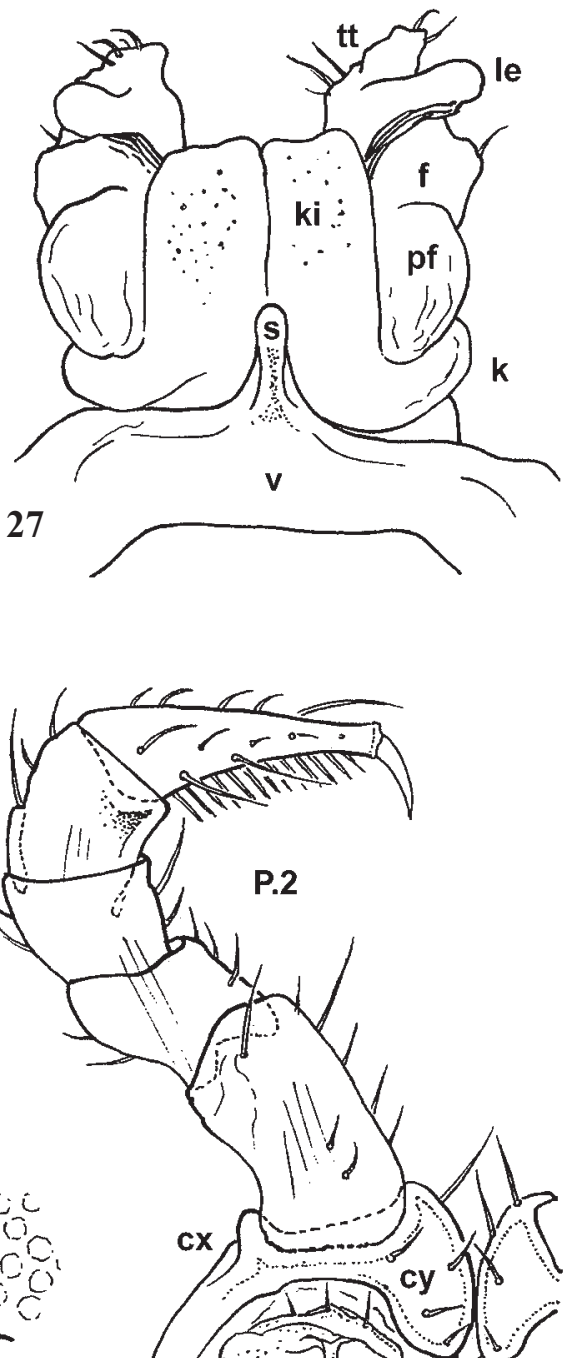

32

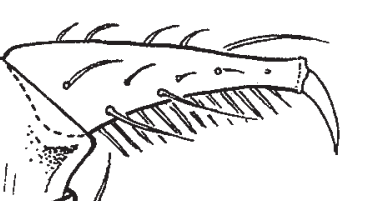

P.2

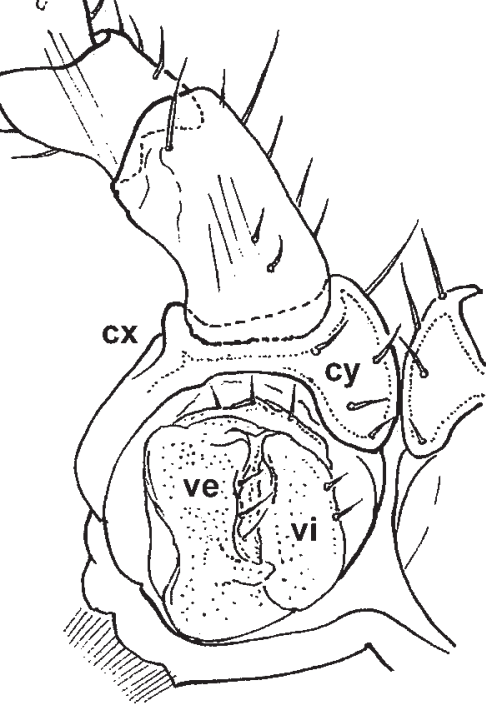

31

30

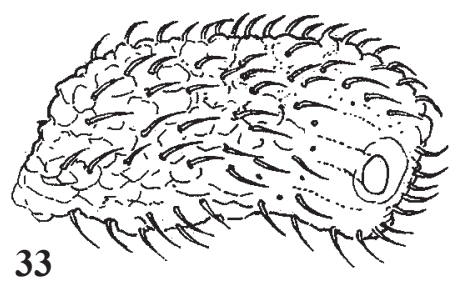

29,31

$0,10 \mathrm{~mm}$

Fig. 26-33. Ebenostenus iberoniger gen.n., sp.n., $\sigma^{\top}$ holotype, $\sigma^{\top}$ et 9 paratypes : 26 - gonopodes (P.9) du $\sigma^{\top}$ holotype, face caudale ; 27 — les mêmes, face orale ; 28 — paragonopodes (P.10) du O holotype, face caudale ; 29 — extrémité du membre gauche du même, grossi ; 30 - paragonopodes (P.10) du $\sigma^{\Upsilon 7}$ holotype, face orale ; 31 — face caudale de la P.2 et de la vulve du côté droit d'une $q$ paratype ; 32 - vue caudale de la paranota droite du $21^{\text {ème }}$ anneau d'un $\sigma^{7}$ paratype ; 33 - extrémité de la même en vue distale.

Fig. 26-33. Ebenostenus iberoniger gen.n., sp.n., $\sigma^{7}$ holotype, $\sigma^{7}$ and $q$ paratypes: 26 - gonopods (legs 9 ) of $\sigma^{7}$ holotype, posterior view; 27 - same, anterior view; 28 - paragonopods (legs 10) of $\sigma^{7}$ holotype, posterior view; 29 - extremity of left member of same, enlarged; 30 - paragonopods (legs 10) of $\sigma^{7}$ holotype, anterior view; 31 - caudal face of leg 2 and of right vulva in + paratype; 32 - caudal view of right paratergum on segment 21 of a $\sigma^{7}$ paratype; 33 - extremity of same in distal view.

Рис. 26-33. Ebenostenus iberoniger gen.n., sp.n., голотип $\sigma^{7}$, паратипы $\sigma^{7}$ и $9: 26$ - гоноподы (ноги 9) голотипа $\sigma^{7}$, сзади; 27 то же, спереди; 28 - парагоноподы (ноги 10) голотипа $\sigma^{7}$, сзади; 29 - вершина левой ноги парагонопода, увеличено; 30 парагоноподы (ноги 10) голотипа $\sigma^{7}$, спереди; 31 - вид сзади ноги 2 и правой вульвы у паратипа $+; 32$ - задний вид правого паратергита сегмента 21 у паратипа $\sigma^{7} ; 33$ - вершина его же в дистальном аспекте. 
annexe interne (ki) en rectangle deux fois plus long que large, chacune étant accolée à son homologue dans le plan sagittal.

Gonopodes postérieurs (fig. 28-30) offrant une structure moins conforme à celle des autres espèces de la sous-famille, bien que chaque membre présente le double coude habituel dans la famille : le sternite en bandeau (v) porte une paire de membres constitués de 6 podomères relativement robustes, dont le plus large est la hanche $(\mathrm{k})$, subtriangulaire et à contour distal en arcature. Le préfémur (pf) s'articule sur le côté de la hanche et comme le fémur (f) s'articule sur le côté du préfémur, il constitue le coude externe de l'arcature du membre. Les trois podomères distaux, situés au-delà du coude, tibia (ti), le plus épais, tarse (ta) et métatarse (mt) le plus petit, sont vaguement globuleux et se dirigent vers l'intérieur. Le métatarse, plus ou moins soudé au tarse, est orné de quelques courtes épines et touche le plan sagittal ; il se prolonge par un uncus $(\mathrm{u})$ transformé en une fine tige rectiligne qui s'incline vers l'extérieur et l'arrière. Cette tige se fissure dans son quart distal en deux tiges de longueur inégale qui ne s'écartent pas l'une de l'autre et qui, toutes les deux, voient leur extrémité s'élargir en deux pointes acérées et opposées l'une à l'autre (fig. 29).

Caractères sexuels des femelles. Peu différents de ceux de Dolistenus, les télopodites des P.1 et des P.2 ont des hanches subtriangulaires (coxosternites) et assez volumineuses. Celles des P.2 recèlent chacune une vulve. Leurs métatarses sont aussi pourvus de « peignes » (6 dents aux P.1, 13 aux P.2) et d'un uncus simple (fig. 31). Les vulves sont de forme vaguement parallélépipédiques, paraissant légèrement plus longues que larges en vue caudale et leurs valves (ve \& vi) sont dissymétriques. Chaque vulve (fig. 31) est encastrée dans la cavité ménagée sur la face caudale de chaque coxosternite et protégée par un épaississement du rebord distal de la cavité qui s'élargit fortement en une protection parasagittale (cy) qui n'existe pas chez D. iberoalbus sp.n.

\section{Conclusion}

Le matériel étudié ici a été récolté dans une aire qui ne représente environ que le quart de celle prospectée par nos collègues (comparer notre carte, fig. 34, à la fig. 14 de Mauriès [2015]). Il est vrai qu'il ne concerne que deux des plus petits ordres de diplopodes, ce qui ne l'empêche pas d'être du plus grand intérêt : parmi seulement les 3 taxa de rang spécifique identifiés, deux sont des espèces nouvelles; l'une appartient à un genre

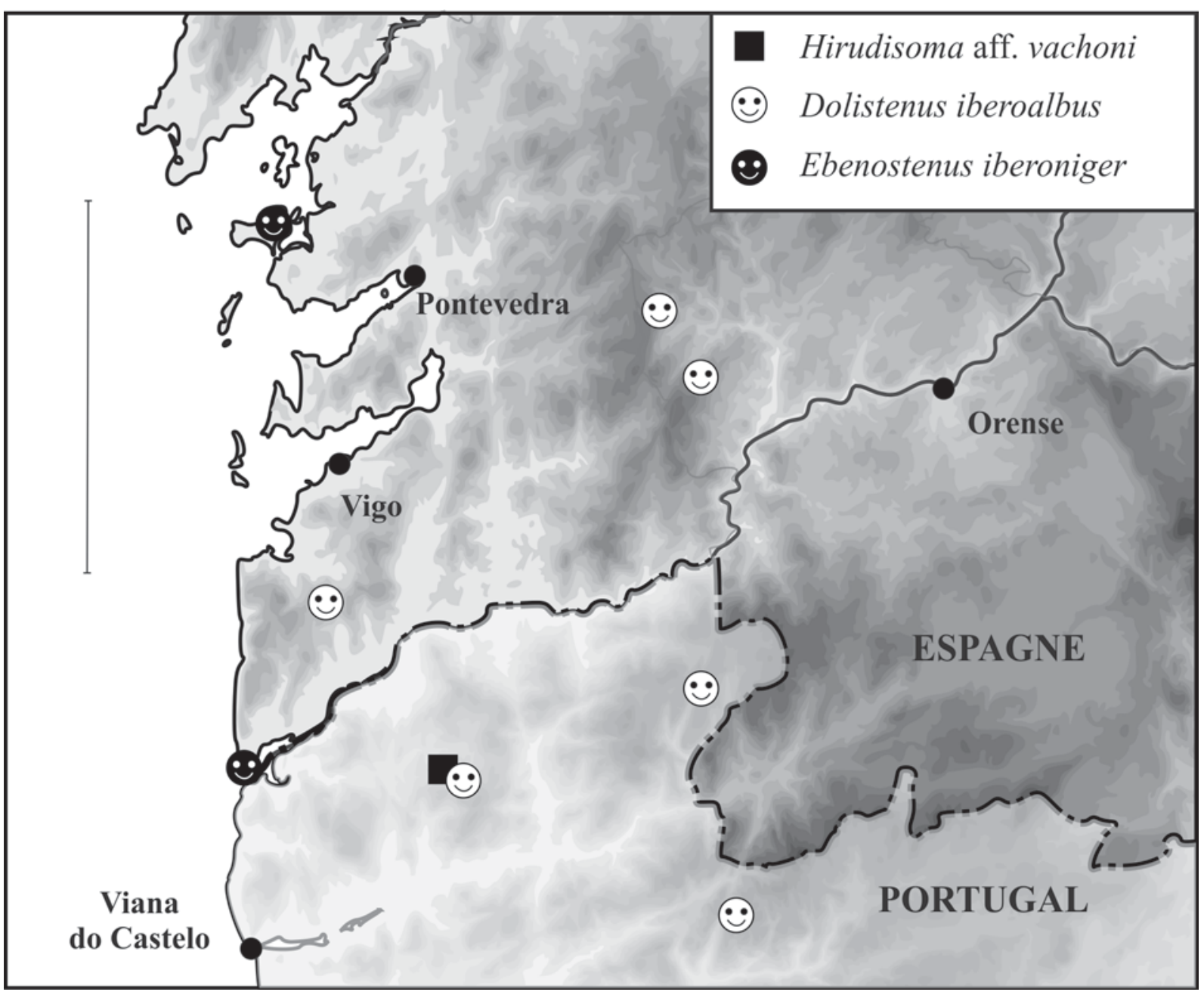

Fig. 34. Répartition géographique des polyzonides et platydesmides de la zone prospectée par les missions britanniques. Echelle : $50 \mathrm{~km}$. Fig. 34. Geographical distribution of polyzoniidans and platydesmidans in the zone prospected by the British expeditions. Scale $50 \mathrm{~km}$. Pис. 34. Геогафическое распространение Polyzoniida и Platydesmida в зоне сборов британских экспедиций. Масштаб 50 км. 
qui est nouveau pour la Péninsule Ibérique, l'autre, qui paraît inféodée aux milieux littoraux, est l'unique espèce d' un genre nouveau pour la science qui, dans la tribu des Dolistenini se singularise de manière frappante par son habitus (double métazonite et ozopores saillants) et sa pigmentation noire.

Avant les missions britanniques, cette partie de la péninsule ibérique était pratiquement inconnue du point de vue diplopodologique. Leurs récoltes ont fait l'objet de plusieurs publications, Mauriès [2005] pour les Glomerida, Read [2007] pour les Julida, Mauriès [2015] pour les Chordeumatida/Craspedosomatida. Tous les groupes n'ont pas encore été étudiés ni publiés ; cependant, on trouvera un certain nombre de données inédites (dont certaines identifications de l'auteur de ces lignes) sous la forme des points bleus des cartes du magnifique Atlas de Kime et Enghoff [2011].

Il est clair que toutes ces découvertes mettent à nouveau en évidence le taux élevé de biodiversité de cette région. Ce qui laisse entrevoir la somme de ce qu'il reste à décrire et ouvre de larges perspectives pour nos collègues espagnols et portugais.

En outre, ce travail de prospection s'inscrit naturellement dans le cadre mis en place par nos collègues espagnols, sous l'égide du Consejo Superior de Investigaciones Cientificas, dans le but de réaliser à long terme un inventaire exhaustif de la faune ibérique.

REMERCIEMENTS. L'auteur est très reconnaissant à nos collègues du British Myriapod and Isopod Group (Steve J. Gregory, R. Desmond Kime, Paul Lee, John Lewis, Helen Read \& J. Paul Richards) ainsi qu'à David Bilton de lui avoir confié ce précieux et si intéressant matériel, et les remercie chaleureusement d'avoir eu la patience d'attendre des résultats qui ont été longs à se réaliser. Il remercie également José Domingo Gilgado, de l'Université d'Alcalà, et son élève Enrique Ledesma qui ont apporté leur pierre au présent travail. Mention spéciale à notre collègue et ami Didier VandenSpiegel (Musée Royal de l'Afrique Centrale, Tervuren, Belgique) qui, avec l'aide logistique de Jonathan Brecko, de la même institution, a pris les photos en couleurs. Et surtout à Sergei Golovatch, visiteur régulier de notre Muséum qui, avec son enthousiasme toujours aussi communicatif et stimulant, et ses remarques avisées, nous accueille généreusement dans les colonnes du Russian Entomological Journal!

\section{Annexe : note nomenclaturale}

Les auteurs du Nomenclator III [Shelley et Golovatch, 2015] ont attiré l'attention sur deux noms de genre, Romanosoma Ceuca, 1967 et Cyrnosoma Mauriès, 1969, qui n'ont pas d'existence légale du fait qu'aucune espèce-type n'avait, par négligence ou par oubli, été désignée par leurs descripteurs. Romanosoma (qui en compte 4 aujourd'hui), a été décrit sur 3 espèces de Roumanie, et Cyrnosoma sur 3 espèces de Corse. Toutes ont été décrites de manière si satisfaisante qu'elles ne méritaient pas d'être enfouies dans les oubliettes de la nomenclature. Pour remédier à cette situation absurde, sont désignées ici pour valider ces deux genres, comme espèces types : pour Romanosoma, Romanosoma cavernicola Ceuca, 1967 et, pour Cyrnosoma, Neo- atractosoma (Cyrnosoma) beroni Mauriès, 1969. Le nom du groupe-famille Cyrnosomatidae se trouve par conséquent lui aussi validé

\section{Références}

Ceuca T. 1967. Quelques autres Diplopodes nouveaux de la faune de la Roumanie // Studia Universitațea Babeş-Bolyai, Series Biologia, Cluj. T.12. Fasc.1. P.107-117.

Fanzago F. 1875. Miriapodi della Calabria // Atti della Società Veneto-trentina di Scienze naturali, Padova. T.4. P.44-76.

Fanzago F. 1881. Miriapodi // Cavanna G..Escursione in Calabria (1877-78). Bullettino della Società Entomologica Italiana. T.12. No.4. P.256-277 (daté 1880).

Golovatch S.I., Evsyukov A., Reip H. 2015. Colobognatha millipedes in the Caucasus (Diplopoda: Polyzoniida, Platydesmida, Siphonocryptida) // Zootaxa. No.3972. No.2. P.250-266.

Hoffman R.L. 1980. Classification of the Diplopoda // Muséum d'histoire naturelle, Genève. 237 pp. (daté 1979).

Kime R.D., Enghoff H. 2011. Atlas of European millipedes (Class Diplopoda). Orders Polyxenida, Glomerida, Platydesmida, Siphonocryptida, Polyzoniida, Callipodida, Polydesmida // Fauna Europaea Evertebrata. No.3. Sofia-Moscow: Pensoft. Vol.1. 282 pp.

Manfredi P. 1933. Dolistenus Menozzii n. sp. della famiglia Platydesmidae (Miriapodi colobognati); e altri Miriapodi della Calabria // Atti della Società Italiana di Scienze Naturali. T.72. P.268-274.

Mauriès J.-P. 1964. Sur quelques Diplopodes de la Péninsule Ibérique // Bulletin de la Société d'Histoire naturelle de Toulouse. T.99. Fasc.1-2. P.157-170.

Mauriès J.-P. 1969. Contribution à la faune épigée et cavernicole de Corse : Diplopodes récoltés par P. Beron // Annales de Spéléologie. T.24. Fasc.3. P.505-527.

Mauriès J.-P. 2005. Essai de classification des Glomerida (Diplopoda) avec description de deux genres nouveaux du nord-ouest de la Péninsule Ibérique // Arthropoda Selecta. Vol.14. No.3. P.241-249.

Mauriès J.-P. 2015. Taxa nouveaux de Diplopodes Craspedosomatides collectés dans le Nord-ouest de la Péninsule Ibérique par les missions britanniques de 1993 et 2004 (Diplopoda, Craspedosomatida) // Bulletin de la Société d'Histoire naturelle de Toulouse. T.150. P.27-57 (daté 2014).

Mauriès J.-P., Barraqueta P. 1985. Myriapodes Diplopodes épigés nouveaux et peu connus de la province de Viscaye (Espagne) ; Vascanthogona et Krauseuma, genres nouveaux de Craspédosomides // Bulletin du Muséum National d'Histoire Naturelle, Paris. Série 4. T.7. Section A. No.2. P.433-449.

Minelli A. 1976. On the genera Dolistenus Fanzago 1874 and Bericostenus Verhoeff 1926 // Fragmenta Entomologica, Roma. T.12. Fasc.2. P.173-181.

Read H.J. 2007. The millipede genus Cylindroiulus Verhoeff, 1894 in north-west Spain and northern Portugal: recent records and descriptions of four new species (Diplopoda, Julida, Julidae) // Graellsia. T.63. P.279-294.

Shelley R.M. 2003. A revised, annotated, family-level classification of the Diplopoda // Arthropoda Selecta. Vol.11. No.3. P.187-207.

Shelley R.M., Golovatch S.I. 2015. Nomenclator Generum et Familiarum Dipopodorum III. A list of the genus-, family-, and ordinal-group names proposed in the Class Diplopoda from 1 January - 31 December 2014 // Arthropoda Selecta. Vol.24. No.1. P.1-26.

Verhoeff K.W. 1926. Über einige südalpine Chilognathen (102. Diplopoden-Aufsatz) // Zoologischer Anzeiger. Bd.66. Nr.1-4. S.65-81.

Verhoeff K.W. 1930a. Über Diplopoden aus Italien, namentlich Piemont. 114. Diplopoden-Aufsatz // Zoologische Jahrbücher, Abteilung für Systematik, Ökologie und Geographie der Tiere. Bd.59. S.387-446.

Verhoeff K.W. 1930b. Zur Geographie, Ökologie und Systematik südalpenländischer Chilognathen (116. Diplopoden-Aufsatz) // Zeitschrift für Morphologie und Ökologie der Tiere. Bd.18. H.4. S.575-668. 